\title{
Robust hybrid estimation and rejection of multi-frequency signals
}

\author{
D. Carnevale ${ }^{1 *}$, S. Galeani ${ }^{1}$ and M. Sassano ${ }^{1}$ and A. Astolfi ${ }^{1,2}$ \\ ${ }^{1}$ Dipartimento di Ing. Civile e Ing. Informatica, Università di Roma "Tor Vergata", 00133 Roma, Italy. \\ ${ }^{2}$ Department of Electrical and Electronic Engineering, Imperial College London, SW7 2AZ, United Kingdom.
}

\begin{abstract}
SUMMARY
We consider the problem of output regulation for LTI systems in the presence of unknown exosystems. The only a priori knowledge about the exosystem consists in the fact that it generates multi-frequency signals, while the number and the value of the frequencies are not known. The control scheme relies on two main components: an estimation algorithm, to reconstruct the signal generated by the exosystem, and a controller, to enforce the output regulation property to the closed-loop system. To tackle the first task, we propose a hybrid observer for the estimation of the (possibly piece-wise continuous) number and values of the frequencies contained in the exogenous signal. The hybrid observer is particularly appealing for numerical implementations and it is combined with a self-tuning algorithm of the free parameters (gains), thus improving its performance even in case of noisy measurements. Semi-global exponential convergence of the estimation error is provided. As far as the second task is concerned, a robust hybrid regulator is designed for practical rejection of the multi-frequency disturbance signal acting on the plant. The result is achieved by exploiting the frequencies estimated by the hybrid observer. The effectiveness of the proposed control scheme is shown by means of numerical simulations. Copyright (c) 0000 John Wiley \& Sons, Ltd.
\end{abstract}

Received ...

KEY WORDS: frequency estimation, robust adaptive control, output regulation, disturbance rejection, hybrid observer.

\section{INTRODUCTION}

The problem of output regulation, which includes as special cases both tracking of references as well as rejection of disturbances, is a classical and fundamental problem in control theory. Typically the reference/disturbance signals are not directly measured but it is a priori assumed that they belong to a pre-specified class of exogenous signals. This is achieved by considering those signals that are generated by a finite dimensional (known) exosystem, the initial condition of such exosystem being the only unknown data. In the case of linear time-invariant systems, solutions to this classical

\footnotetext{
*Correspondence to: Dipartimento di Ing. Civile e Ing. Informatica, Università di Roma "Tor Vergata", 00133 Roma, Italy. E-mail: daniele.carnevale@uniroma2.it

Contract/grant sponsor: ENEA-EUROFUSION

Copyright (C) 0000 John Wiley \& Sons, Ltd.

Prepared using acsauth.cls [Version: 2010/03/27 v2.00]
} 
problem have been proposed both in the geometric setting $[1,2]$ and in the algebraic setting $[3,4,5]$, just to name a few.

When the exosystem, on the other hand, is not perfectly known, the problem becomes much more complicated since it is necessary either to directly adapt the internal model of the exosystem contained in the regulator (see for instance $[6,7,8]$, often considering the case of nonlinear system), or to identify the frequency characteristics of the exogenous signals entering the plant and then either redesign the regulator according to the obtained information or directly cancel the exogenous disturbances using the estimated signal (see for instance $[9,10,11,12,13,14,15]$ ). The latter approach is pursued here.

In this paper the multi-frequency hybrid estimator introduced in $[16,17]$ is combined with the robust practical internal-model based controller in [18] to solve the problem of output regulation in the presence of unknown exosystems. In particular, we suppose that the only knowledge about the structure of the exosystem consists in the fact it generates signals obtained as linear combination of sinusoids at different frequencies.

The problem of finding the unknown angular frequencies $\omega_{i}$ 's of a signal of the form

$$
y(t)=\sum_{i=1}^{n} E_{i} \sin \left(\omega_{i} t+\phi_{i}\right),
$$

where also $E_{i}$ 's and $\phi_{i}$ 's are unknown, has been extensively addressed using classical Fourier analysis of batch data [19], on-line methods based on notch filters [20] and Kalman filters [21], adaptive schemes [22] and adaptive identifiers [23, 24, 25], filtered transformations [26], Immersion and Invariance techniques [27], and hybrid systems [16] just to name a few. We propose an algorithm to estimate the number $n$ and the values of the frequencies in (1) and, in addition, to autonomously adjust the (re-)sampling time $T$ of the signal (1) and the gains of the observer, thus providing a self-tuning hybrid observer with enhanced performance with respect to [16]. Despite the fact that the selection of the gains in [16] (as well as in [27]) are crucial to obtain satisfactory performances, this choice is delegated, as it typically happens in the vast majority of the approaches, to the user. Moreover, as pointed out in Section 2.2, a suitable selection of such gains is strongly related to the actual value of the frequencies $\omega_{i}$ 's, hence making their a priori choice almost impossible. More specifically, the observer in [16] requires the selection of the sampling time $T$ to collect a given amount of samples of (1) which are processed to obtain the estimates of the $\omega_{i}$ 's. The exponential convergence of the estimation error $\omega-\hat{\omega}$ to zero has been seen to critically depend on the selection of $T$, which may not be correctly selected, hence hindering the convergence properties of the estimates (see Lemma 1). The objective herein is to modify the hybrid observer proposed in [16] in such a way that the correct value of the number $n$ and a suitable choice of the sampling time $T$ are computed on-line by processing a batch of collected measurements. With respect to previous work, we further assume that the number of frequencies $n$ of the signal (1) is not known, as in [28] and [29], and it can vary over time, namely $n(t)$ may be an unknown piece-wise constant signal.

Then, we combine the proposed observer with an adaptive hybrid controller to solve the problem of output regulation of linear systems in the presence of disturbances (references) produced by frequency generators of unknown dimension and parameters. The controller consists of three main components. In addition to the multi-frequency identifier described above, we design a residual 
generator, which provides a signal suitable for the identification of the frequencies contained in the exogenous signal, and a servocompensator, which contains an internal model of the (identified) frequencies combined with a stabilizing compensator.

The rest of the paper is structured as follows. To begin with, in Section 2, the hybrid observer for the estimation of the unknown frequencies in a signal of the form of (1) is introduced and discussed. The section is concluded by defining a self-tuning algorithm for the gains (free parameters) of the observer, which constitutes the main tool employed towards the solution of the output regulation problem in the presence of unknown exosystems, which is formalized in Section 3. The architecture of the proposed control scheme and its main components and features are described in the same section. Finally a case study is shown in Section 5 to substantiate the effectiveness of the method, while conclusions are drawn in Section 6.

\section{HYBRID OBSERVER FOR FREQUENCY ESTIMATION}

In this section we propose a hybrid observer to estimate the (unknown) frequencies contained in the signal (1), regardless of the unknown values of $E_{i}$ 's and $\phi_{i}$ 's. The observer is then combined with an algorithm that permits the autonomous adjustment of the design parameters.

\subsection{Hybrid observer design}

The signal (1) can be seen as the output $y(t)=C_{\omega} x(t)$ of the linear time-invariant system described by the equations

$$
\dot{x}=A_{\omega} x=\operatorname{diag}\left\{\left[\begin{array}{cc}
0 & \omega_{i} \\
-\omega_{i} & 0
\end{array}\right]\right\} x, i=1, \ldots, n,
$$

where $x \in \mathbb{R}^{2 n}, C_{\omega}=[0,1,0,1,0, \ldots, 1]$, with unknown initial condition $x(0)$ and $\omega_{i}$ 's. We suppose that the signal (1) consists of, at most, $N$ frequencies, namely $n \leq N$, with $N$ known a priori. The signal $y(t)$ is (re-)sampled with sampling time $T=p T_{s}$, for some $p \in \mathbb{N}_{\geq 1}$ and the hardware sampling time $T_{s}$. Notice that the hardware sampling time $T_{s}$ limits, by Nyquist-Shannon's Theorem, the higher angular frequency that can be reconstructed to $\omega_{\max }:=\pi / T_{s}$. In the following, we assume that the frequencies in (2) are upper-bounded by $\bar{\omega}<\omega_{\max }$, namely $\omega_{i} \leq \bar{\omega}$ for all $i=1, \ldots, n$. The value of $\bar{\omega}$ induces in turns an upper-bound on the largest sampling time that can be considered, namely enforcing $T=p T_{s}<\pi / \bar{\omega}$. The measurements vector is defined as

$$
Y_{k}:=\left[\begin{array}{c}
y\left(t_{k-2 n}\right) \\
\cdots \\
y\left(t_{k-2}\right) \\
y\left(t_{k-1}\right)
\end{array}\right]=O x\left(t_{k-2 n}\right),
$$


where $t_{k}=k T$, i.e. $y\left(t_{k}\right)=y\left(k p T_{s}\right)$, and

$$
O:=\left[\begin{array}{c}
C_{\omega} \\
C_{\omega} A_{D} \\
\cdots \\
C_{\omega} A_{D}^{2 n-1}
\end{array}\right], \quad A_{D}:=e^{A_{\omega} T} .
$$

The characteristic polynomial of $A_{D}$ is

$$
\begin{aligned}
& p_{A_{D}}(\lambda)=\prod_{i=1}^{n}\left(\lambda^{2}-2 \cos \left(\omega_{i} T\right) \lambda+1\right) \\
& =\lambda^{2 n}+a_{2 n-1} \lambda^{2 n-1}+\cdots+a_{1} \lambda+1,
\end{aligned}
$$

with symmetric coefficients, i.e. such that $a_{2 n-h}=a_{h}, h=1, \ldots, n-1$. The coefficients $a_{i}$ of $p_{A_{D}}(\lambda)$ can be compactly expressed as

$$
a:=\left[\begin{array}{c}
1 \\
a_{1} \\
a_{2} \\
\vdots \\
a_{2 n-2} \\
a_{2 n-1}
\end{array}\right]=\left[\begin{array}{cc}
1 & 0 \\
0 & S
\end{array}\right]\left[\begin{array}{c}
1 \\
a_{1} \\
a_{2} \\
\vdots \\
a_{n-1} \\
a_{n}
\end{array}\right]=\hat{S}\left[\begin{array}{c}
1 \\
a_{c}
\end{array}\right]
$$

where $S_{i}$ is the $i$-th row of $S \in \mathbb{R}^{2 n-1 \times n}$ and the matrices $S, \hat{S} \in \mathbb{R}^{2 n \times n+1}$ and the coefficients vector $a_{c} \in \mathbb{R}^{n}$ are defined according to

$$
\begin{aligned}
S_{i} & := \begin{cases}1 & \text { if } i=j \text { or } 2 n-i=j, \\
0 & \text { otherwise, }\end{cases} \\
\hat{S} & =\left[\begin{array}{ll}
1 & 0 \\
0 & S
\end{array}\right], \\
a_{c, i} & =a_{i}=f_{i}(\omega), \quad i=1, \ldots, n,
\end{aligned}
$$

where $f_{i}(\omega)=\sum(-2)^{i} \mathcal{Q}_{j}^{i}(\omega)$ is the sum of the $\left(\begin{array}{c}n \\ i\end{array}\right)$ monomials $\mathcal{Q}_{j}^{i}(\omega), j=1, \ldots,\left(\begin{array}{c}n \\ i\end{array}\right)$, with $\mathcal{Q}_{j}^{i}(\omega)$ obtained as the product of the elements of the $j$-th combination without repetition of $i$ elements of the set $\left\{\cos \left(\omega_{1} T\right), \ldots, \cos \left(\omega_{n} T\right)\right\}$. Equivalently, the $f_{i}(\omega)$ corresponds to the coefficients of the characteristic polynomial of $A_{D}$ in (5) for $i=1, \ldots, n$. The above definitions $\tilde{Y}_{k}=\left[y\left(t_{k-2 n-1}\right), \ldots, y\left(t_{k-1}\right)\right]^{\prime} \in \mathbb{R}^{2 n-1}$ allow to write

$$
y\left(t_{k}\right)=-Y_{k}^{\prime} a=-Y_{k}^{\prime} \hat{S}\left[\begin{array}{c}
1 \\
a_{c}
\end{array}\right]=-y\left(t_{k-2 n}\right)-\tilde{Y}_{k}^{\prime} S f(\omega),
$$


and

$$
Y_{k+1}=-\left[\begin{array}{ccc}
y\left(t_{k-4 n+1}\right) & \cdots & y\left(t_{k-2 n}\right) \\
\vdots & & \vdots \\
y\left(t_{k-2 n-1}\right) & \cdots & y\left(t_{k-2}\right) \\
y\left(t_{k-2 n}\right) & \cdots & y\left(t_{k-1}\right)
\end{array}\right] a=:-\bar{Y}_{k} a .
$$

It can be easily proven, by observability of the system (2), that $\operatorname{rank}\left(\bar{Y}_{k}\right)=2 n$ for all $T \neq 2 \pi / \omega_{i}$ with $i=1, \ldots, n$. Then, the vector $a$ can be readily evaluated by

$$
a=-\bar{Y}_{k}^{-1} Y_{k+1},
$$

collecting the first $4 n$ data to fill the matrix $\bar{Y}_{k}$. This is a classical approach to obtain the parameters $a_{i}$ in finite time. It is important to note that the numerical invertibility of the matrix $\bar{Y}_{k}$ critically depends on the sampling time $T$. In fact, if $T$ is too small with respect to $\min _{i}\left\{2 \pi / \omega_{i}\right\}$ the matrix $\bar{Y}_{k}$ is ill-conditioned.

To obtain the estimate of $a_{c}$ it is possible to define $\bar{Y}_{k}^{c} \in \mathbb{R}^{2 n \times 2 n-1}$ such that

$$
\bar{Y}_{k}=\left[Y_{k-2 n-1}, Y_{k-2 n}, \ldots, Y_{k}\right] \triangleq\left[Y_{k-2 n-1}, \bar{Y}_{k}^{c}\right]
$$

and then

$$
a_{c}=-\left(S^{\prime} \bar{Y}_{k}^{c \prime} \bar{Y}_{k}^{c} S\right)^{-1} S^{\prime} \bar{Y}_{k}^{c \prime}\left(Y_{k+1}+Y_{k-2 n-1}\right) .
$$

Note that the matrix $S^{\prime} \bar{Y}_{k}^{c \prime} \bar{Y}_{k}^{c} S$ is invertible if $\operatorname{rank}\left(\bar{Y}_{k}\right)=2 n$. As usual, to render this method less sensitive to measurement noise, it is possible to consider more than $4 n$ samples of the output, stacked within the (tall) matrix $\bar{Y}_{k}$ and to obtain estimates of $a$ or $a_{c}$ by exploiting the Moore-Penrose pseudoinverse of $\bar{Y}_{k}$.

\section{Remark 1}

The $\omega_{i}$ 's can be obtained from the $a_{i}$ 's by finding the zeros $\zeta_{i} \pm j \sqrt{1-\zeta_{i}^{2}} \in(-1,1)$ of the $2 n$ order polynomial $\Pi_{i=1}^{n}\left(\lambda^{2}-2 \zeta_{i} \lambda+1\right)$ with $\zeta_{i}=\cos \left(\omega_{i} T\right)$ and, assuming $0<\omega_{i} T<\pi, \omega_{i}=$ $\arccos \left(\zeta_{i}\right) / T$. Namely, it is sufficient to run a root-finding algorithm on the polynomial (5) with $\lambda=e^{j \alpha}$ and $\alpha \in(0,2 \pi)$.

We now consider the hybrid observer $\mathcal{H}$ introduced in [16], which is designed for the direct estimation of $\omega=\left[\omega_{1}, \ldots, \omega_{n}\right]^{\prime}$ by employing the inverse of the Jacobian matrix $\partial f(\hat{\omega}) / \partial \hat{\omega}$. In the following, we additionally propose an alternative approach that does not rely on the Jacobian matrix inversion. Nonetheless, the use of the Jacobian matrix inverse allows us to provide a simpler, 
somewhat more explanatory, proof of the result (not reported in [16]). Towards this end, define

$$
A_{0}=\left[\begin{array}{ccccc}
0 & 1 & 0 & \cdots & 0 \\
0 & 0 & 1 & \ddots & \vdots \\
\vdots & & \ddots & \ddots & 0 \\
0 & \cdots & \cdots & 0 & 1 \\
0 & \cdots & \cdots & \cdots & 0
\end{array}\right], \quad B_{0}=\left[\begin{array}{c}
0 \\
\vdots \\
\vdots \\
0 \\
1
\end{array}\right]
$$

with the shifting matrix $A_{0} \in \mathbb{R}^{2 n \times 2 n}, B_{0} \in \mathbb{R}^{2 n}$. Then the flow and jump maps of $\mathcal{H}$ with state

$$
\xi=\left[\begin{array}{llll}
\hat{\omega}^{\prime} & \zeta^{\prime} & \chi & \tau
\end{array}\right]^{\prime} \in \mathcal{O}
$$

where $\hat{\omega} \in \mathbb{R}^{n}, \zeta \in \mathbb{R}^{2 n}, \chi \in \mathbb{R}, \tau \in \mathbb{R}$, are

$$
\left.\begin{array}{rl}
\dot{\hat{\omega}} & =-\gamma\left(\frac{\partial f(\hat{\omega})}{\partial \hat{\omega}}\right)^{-1}\left[0, S^{\prime}\right] \zeta e, \\
\dot{\zeta} & =0 \\
\dot{\chi} & =0 \\
\dot{\tau} & =1 \\
\hat{\omega}^{+} & =\hat{\omega} \\
\zeta^{+} & =A_{0} \zeta+B_{0} \chi, \\
\chi^{+} & =y \\
\tau^{+} & =0
\end{array}\right\} \text { if } \xi \in \mathcal{C}
$$

where $\gamma>0$ is the observer gain, and the error $e$ is given by

$$
e(t, k)=y\left(t_{k}\right)+y\left(t_{k-2 n}\right)+\tilde{Y}_{k}^{\prime} S f(\hat{\omega}(t, k)) .
$$

The flow set $\mathcal{C}$ and the jump set $\mathcal{D}$ are defined as

$$
\begin{aligned}
& \mathcal{C} \triangleq\{\xi \in \mathcal{O}: \tau \in[0, T]\}, \\
& \mathcal{D} \triangleq\{\xi \in \mathcal{O}: \tau \geq T\} .
\end{aligned}
$$

The vector $\zeta$ maintains the past $2 n$ values of the input $y$, i.e. $Y_{k}=\zeta(t, k)$ whereas $\chi(t, k)=y\left(t_{k}\right)$ for all $t \in\left[t_{k}, t_{k+1}\right)$. Note that $2 n$ samples of $y$ have to be collected in $\zeta(0,0)$ before the estimation process can be started. By definition of the jump and the flow sets, the observer (discrete-time) resets are triggered every $T$ seconds. The hybrid formulation proposed in [30] allows to easily take into account jumps at sampling times as well as continuous dynamics between two consecutive jumps. Before presenting the main result of this section that summarizes the convergence properties of the observer $\mathcal{H}$, we consider the following standing assumption.

\section{Assumption 1}

The parameters of the signal (1) satisfy $E_{i} \neq 0,0<\omega_{i}<\pi / T_{s}$ and $\omega_{i} \neq \omega_{j}$ for any $i \neq j$ with $(i, j) \in\{1, \ldots, n\}$. 


\section{Remark 2}

The implementation of (14b) requires the invertibility of the Jacobian matrix $\partial f(\hat{\omega}) / \partial \hat{\omega}$, which can be shown to hold if and only if all the elements of $\hat{\omega}$ are pairwise distinct and different from zero. Such a condition can be enforced by modifying the flow dynamics of $\hat{\omega}$ in (14b) by introducing a barrier function defined as follows:

$$
\dot{\hat{\omega}}=-\gamma\left(\frac{\partial f(\hat{\omega})}{\partial \hat{\omega}}\right)^{-1}\left(\left[0, S^{\prime}\right] \zeta e+\alpha(\hat{\omega})\right)
$$

where the $i$-th component $\alpha_{i}(\hat{\omega})$ of $\alpha(\hat{\omega})$ is given by

$$
\alpha_{i}(\hat{\omega})=\max \left\{1, \frac{\varepsilon}{\hat{\omega}_{i}-\hat{\omega}_{i-1}}\right\}-\max \left\{1, \frac{\varepsilon}{\hat{\omega}_{i+1}-\hat{\omega}_{i}}\right\}
$$

$i=1, \ldots, n$, with $\varepsilon>0, \hat{\omega}_{0}=0$ and $\hat{\omega}_{n+1}=\frac{\pi}{T}$, and initializing $\hat{\omega}$ so that $0<\hat{\omega}_{1}(0,0)<\hat{\omega}_{2}(0,0)<$ $\cdots<\hat{\omega}_{n}(0,0)<\frac{\pi}{T}$. Under the assumption that the actual frequencies in $\omega$ are separated by (at least) $\varepsilon>0$, the desired equilibrium $\hat{\omega}=\omega$ of (14b) is preserved for the system (15), meanwhile singularities of $\partial f(\hat{\omega}) / \partial \hat{\omega}$ are avoided. Furthermore, as anticipated above the Jacobian inversion, which clearly involves numerical issues, can be avoided by replacing the dynamics (14b) (similarly for (15)) with a gradient-like algorithm described by the equations

$$
\dot{\hat{\omega}}=-\gamma\left(\frac{\partial f(\hat{\omega})}{\partial \hat{\omega}}\right)^{\prime}\left(\left[0, S^{\prime}\right] \zeta e+\alpha(\hat{\omega})\right) \text {. }
$$

Note that if the initial conditions of $\hat{\omega}_{i}$ are pair-wise distinct, numerical approximations typically allow to neglect the term $\alpha(\cdot)$, which has been introduced in (15) to avoid singularities of the matrix $\partial f(\hat{\omega}) / \partial \hat{\omega}$.

\section{Theorem 1}

Suppose that Assumption 1 holds and that the Jacobian matrix $\partial f(\hat{\omega}) / \partial \hat{\omega}$ is invertible ${ }^{\dagger}$. Then the estimation error $\tilde{a}(t, k)=\hat{a}(t, k)-a=\left[0,(S(f(\hat{\omega}(t, k))-f(\omega)))^{\prime}\right]^{\prime}$, with $\hat{\omega}(t, k)$ provided by the observer $\mathcal{H}$ with flow map (14b) and jump map (14c), uniformly exponentially converges to zero as $k$ goes to infinity.

Proof: The flow map of $\hat{a}$ accordingly with the dynamics of $\hat{\omega}$ in (14b) is

$$
\dot{\hat{a}}=-\gamma \zeta\left(\chi+\zeta^{\prime} \hat{a}\right)=-\gamma \zeta e,
$$

and the time derivatives of the function $V(k, t)=\|\tilde{a}(k, t)\|^{2} / 2$ are (with $\left.\zeta(k, t)=Y_{k}\right)$

$$
\dot{V}(k, t)=-\gamma \tilde{a}^{\prime}(k, t) Y_{k} Y_{k}^{\prime} \tilde{a}(k, t)
$$

\footnotetext{
${ }^{\dagger}$ As discussed in Remark 2 this assumption may be removed by implementing (15) in place of the first equation of (14b). 
and

$$
\begin{aligned}
\dot{\tilde{a}}(k, t) & =-\gamma Y_{k} Y_{k}^{\prime} \tilde{a}(k, t), \\
\dot{\Phi}_{k}(t) & =-\gamma Y_{k} Y_{k}^{\prime} \Phi_{k}(t), \\
\tilde{a}(k, t) & =\Phi_{k}(t) \tilde{a}\left(k, t_{k}\right), \forall t \in\left[t_{k}, t_{k+1}\right),
\end{aligned}
$$

with $\Phi_{k}(t) \in \mathbb{R}^{2 n \times 2 n}$. It follows that the $2 n-1$-dimensional manifold $\mathcal{M}_{k}$ described by $Y_{k}^{\prime} \tilde{a}=0$ is stable, whereas its attractivity, for fixed $k$, is ensured by classical La-Salle's invariance principle letting $t_{k+1}=\infty$ (or equivalently $\gamma=+\infty$ ), yielding $\tilde{a}(k, \infty) \in \operatorname{Ker}\left(Y_{k} Y_{k}^{\prime}\right)=\operatorname{Im}\left(Y_{k}\right)^{\perp}$. Note that $\tilde{a}(k, t)$ moves along $Y_{k}^{\perp}$ as $t$ grows from $t_{k}$, i.e. , there does not exist a matrix $M \in \mathbb{R}^{2 n \times 2 n}$ such that $\tilde{a}(k, t)=M Y_{k}$ for all $t$ in the interior of $\Delta_{k}:=\left[t_{k}, t_{k+1}\right)$. The integral of $\dot{V}$ over $2 n T$ satisfies

$$
\int_{k T}^{(k+2 n) T} \dot{V} d \tau=-\gamma \sum_{h=0}^{2 n-1} \int_{(k+h) T}^{(k+h+1) T} \tilde{a}^{\prime}(k+h, \tau) Y_{k+h} Y_{k+h}^{\prime} \tilde{a}^{\prime}(k+h, \tau) d \tau .
$$

With $A_{k}:=Y_{k} Y_{k}^{\prime}=A_{k}^{\prime}$ and $\Phi_{k}(t)=e^{-\gamma A_{k} t},(20)$ rewrites into

$$
\begin{aligned}
& \int_{k}^{k+2 n} \dot{V} d \tau=-\gamma \tilde{a}^{\prime}\left(k, t_{k}\right)\left(\int_{0}^{T} \Phi_{k}^{\prime}(\tau) A_{k} \Phi_{k}(\tau)+\right. \\
& \left.\Phi_{k}^{\prime}(T) \Phi_{k+1}^{\prime}(\tau) A_{k+1} \Phi_{k+1}(\tau) \Phi_{k}(T)+\ldots d \tau\right) \tilde{a}\left(k, t_{k}\right) \\
& =-\tilde{a}^{\prime}\left(k, t_{k}\right)\left(c_{0} Y_{k} Y_{k}^{\prime}+c_{1} \Phi_{k}^{\prime}(T) Y_{k+1} Y_{k+1}^{\prime} \Phi_{k}(T)+\ldots\right. \\
& \left.+c_{2 n} \Pi_{h=0}^{2 n-1} \Phi_{k+h}^{\prime}(T) Y_{k+2 n-1} Y_{k+2 n-1}^{\prime} \Pi_{h=2 n-1}^{0} \Phi_{k+h}(T)\right) \tilde{a}\left(k, t_{k}\right),
\end{aligned}
$$

where

$$
c_{h}=\left\|Y_{k+h}\right\|^{2}\left(1-e^{-2 \gamma T}\right) / 2, h=\{0,1, \ldots, 2 n-1\} .
$$

Note that $c_{\max } \geq c_{h} \geq c_{\min }$ since $\left\|Y_{k}\right\|$ is uniformly lower and upper bounded for any $k \geq 2 n$. The rhs of (21a) can be rewritten as

$$
-\tilde{a}^{\prime}\left(k, t_{k}\right) \Lambda_{c} \bar{Y}_{k} \bar{Y}_{k}^{\prime} \tilde{a}\left(k, t_{k}\right)
$$

where

$$
\begin{aligned}
& \bar{Y}_{k}:=\left[\begin{array}{llll}
Y_{k} & \Phi_{k}^{\prime}(T) Y_{k+1} & \ldots & \Pi_{h=0}^{2 n-1} \Phi_{k+h}^{\prime}(T) Y_{k+2 n-1}
\end{array}\right], \\
& \Lambda_{c}=\operatorname{diag}\left\{c_{h}\right\}, h=\{0,1, \ldots, 2 n-1\} .
\end{aligned}
$$

Note that $\Phi_{k}^{\prime}(T)$ is a projection matrix on the space $Y_{k}^{\perp}$, which implies that ${ }^{\ddagger} \Phi_{k}^{\prime}(\infty) v \in \operatorname{Im}\left(Y_{k}\right)^{\perp}$ for any $v \in \mathbb{R}^{2 n}$. To prove that

$$
\bar{Y}_{k}^{\prime} \bar{Y}_{k} \geq \gamma_{1} I
$$

$\bar{\ddagger} \Phi_{k}^{\prime}(\infty)=\left.\right|_{\gamma=+\infty} \Phi_{k}^{\prime}(T)$. 
for some $\gamma_{1}>0$, yielding exponential stability of the origin ${ }^{\S}$ of (19a), first note that $\left\|\Phi_{k}^{\prime}(T) Y_{k}\right\| \geq$ $\left\|\Phi_{k}^{\prime}(\infty) Y_{k}\right\|$, and defining $Y_{k+h, \infty}=\Pi_{h=0}^{2 n-1} \Phi_{k+h}^{\prime}(\infty) Y_{k+h}$, inequality (25) is satisfied if we are able to prove that $\bar{Y}_{k, \infty}^{\prime} \bar{Y}_{k, \infty} \geq \gamma_{1, \infty} I$, for some $\gamma_{1, \infty}>0$, where

$$
\bar{Y}_{k, \infty}^{\prime}=\left[\begin{array}{llll}
Y_{k, \infty} & Y_{k+1, \infty} & \cdots & Y_{k+2 n-1, \infty}
\end{array}\right] .
$$

To conclude, rewriting $\bar{Y}_{k, \infty}$ as

$$
\bar{Y}_{k, \infty}=\operatorname{diag}\left\{\left\|Y_{k, \infty}\right\|\right\}\left[\begin{array}{c}
\eta_{k, \infty}^{\prime} \\
\eta_{k+1, \infty}^{\prime} \\
\vdots \\
\eta_{k+2 n-1, \infty}^{\prime}
\end{array}\right]
$$

with $Y_{k, \infty}^{\prime}=\left\|Y_{k, \infty}\right\| \eta_{k, \infty}$, it follows that $v^{\prime} \bar{Y}_{k, \infty} \bar{Y}_{k, \infty}^{\prime} v \geq \min _{h=0, \ldots, 2 n}\left\|Y_{k, \infty}\right\|\|v\|:=\gamma_{1, \infty}\|v\|$ for any $v \in \mathbb{R}^{2 n}$, given that $\left\|\eta_{k, \infty}\right\|=1, \eta_{k+1, \infty} \in \eta_{k, \infty}^{\perp}$, and $\mathbb{R}^{2 n}=\operatorname{span}\left\{\eta_{k, \infty}, \eta_{k+1, \infty}, \cdot, \eta_{k+2 n-1, \infty}\right\}$ for any $k \geq 2 n$.

\section{Remark 3}

A result similar to Theorem 1 may be obtained with the modified dynamics (16). In particular, the proof follows the same reasoning above, but requires the matrix $\left(\frac{\partial f(\hat{\omega})}{\partial \hat{\omega}}\right)\left(\frac{\partial f(\hat{\omega})}{\partial \hat{\omega}}\right)^{\prime}$ to be full rank, which is in turns ensured by the presence of the repulsive term $\alpha(\cdot)$ when initial estimates $\hat{\omega}(0,0)$ are taken such that $\hat{\omega}_{i}(0,0) \neq \hat{\omega}_{j}(0,0)$ for $i \neq j,(i, j) \in\{1, \ldots, n\}$.

As suggested by (17), it is also possible to estimate the vector $a_{c}$ or $a$ without estimating first the vector of the frequencies $\omega$.

The design parameters of the above observer are the number $n$ of (estimated) frequencies, the gain $\gamma$, and the sampling time $T$, i.e. the value of $p \in \mathbb{N}_{\geq 1}$ such that $T=p T_{s}$. However, the sensitivity of the performance with respect to $\gamma$ is negligible in comparison with the importance of $n$ and $T$, the latter of which critically affects the convergence properties of the algorithm, which are on the other hand significantly enhanced by a correct choice of its value. This aspect constitutes the topic of the following section in which a method to automatically adjust the values of $n$ and $T$ is proposed.

\subsection{On-line computation of the number of frequencies and the sampling time}

To determine the correct number $n$ of frequencies, and consequently the sampling time, we propose to exploit the information provided by the rank of the matrix $\bar{Y}_{k}$. Therefore, the relation between the rank of the matrix $\bar{Y}_{k}$ and the sampling time $T$ is summarized in the following lemma.

\section{Lemma 1}

Assume that $\omega_{i}<\omega_{\max }$ for all $i=1, \ldots, n$ and that $x_{0}$ excites all the modes of (2). Let $\bar{Y}_{k} \in$ $\mathbb{R}^{2 \hat{n} \times 2 \hat{n}}$ be obtained by the samples $y\left(t_{k}\right)$ with sampling time $T$, then

i) $\operatorname{rank}\left(\bar{Y}_{k}\right)=2 \hat{n}$ if $\hat{n} \leq n$ and $T<\pi / \max _{i}\left\{\omega_{i}\right\}$

ii) $\operatorname{rank}\left(\bar{Y}_{k}\right)<2 \hat{n}$ if $\hat{n}=n$ and $T=\pi / \omega_{i}$ for some $i=1, \ldots, n$

\footnotetext{
$\S$ There is no necessity to analyse effects of the jumps on the estimation error since $\tilde{a}\left(k, t_{k+1}\right)=\tilde{a}\left(k+1, t_{k+1}\right)$.
}

T This requirement is equivalent to the condition $E_{i} \neq 0$ for all $i=1, \ldots, n$. 


$$
\begin{aligned}
f(\omega) & =\left[\begin{array}{cc}
-2 \cos \left(\omega_{3} T\right)-2 \cos \left(\omega_{2} T\right)-2 \cos \left(\omega_{1} T\right) \\
3+4 \cos \left(\omega_{3} T\right) \cos \left(\omega_{2} T\right)+4 \cos \left(\omega_{3} T\right) \cos \left(\omega_{1} T\right)+4 \cos \left(\omega_{1} T\right) \cos \left(\omega_{2} T\right) \\
-4 \cos \left(\omega_{2} T\right)-4 \cos \left(\omega_{1} T\right)-4 \cos \left(\omega_{3} T\right)-8 \cos \left(\omega_{3} T\right) \cos \left(\omega_{1} T\right) \cos \left(\omega_{2} T\right)
\end{array}\right] \\
\nabla f(\omega) & =\left[\begin{array}{ccc}
2 \sin \left(\omega_{1} T\right) T & 2 \sin \left(\omega_{2} T\right) T \\
-4 \sin \left(\omega_{1} T\right) T\left(\cos \left(\omega_{3} T\right)+\cos \left(\omega_{2} T\right)\right) & -4 \sin \left(\omega_{2} T\right) T\left(\cos \left(\omega_{3} T\right)+\cos \left(\omega_{1} T\right)\right) & -4 \sin \left(\omega_{3} T\right) T\left(\cos \left(\omega_{2} T\right)+\cos \left(\omega_{1} T\right)\right) \\
4 \sin \left(\omega_{1} T\right) T\left(1+2 \cos \left(\omega_{3} T\right) \cos \left(\omega_{2} T\right)\right) & 4 \sin \left(\omega_{2} T\right) T\left(1+2 \cos \left(\omega_{3} T\right) \cos \left(\omega_{1} T\right)\right) & 4 \sin \left(\omega_{3} T\right) T\left(1+2 \cos \left(\omega_{1} T\right) \cos \left(\omega_{2} T\right)\right)
\end{array}\right]
\end{aligned}
$$

iii) $\operatorname{rank}\left(\bar{Y}_{k}\right)<2 \hat{n}$ if $\hat{n}>n$ and $T<\pi / \max _{i}\left\{\omega_{i}\right\}$.

Proof: Consider first the case with $\hat{n}=n$ and define

$$
\bar{X}_{k}=\left[x\left(t_{k-4 n+1}\right), \ldots, x\left(t_{k-2 n}\right)\right]
$$

Since $x\left(t_{k+1}\right)=A_{D} x\left(t_{k}\right)$, then for $k=4 n-1 \quad$ and $\quad x_{0}=B_{0} \quad$ it holds $\quad \bar{X}_{k}=R=$ $\left[B_{0}, A_{D} B_{0}, \ldots, A_{D}^{2 n} B_{0}\right]$. Since $x_{0}=B_{0}$ excites all the modes of (2) by assumption, the reachability matrix $R$ of $\left(A_{D}, B_{0}\right)$ is full rank if $T \neq T_{i} / 2=\pi / \omega_{i}, i=1, \ldots, n$, induced by the second inequality in $i$ ) and $i i i)$. Trivially for $k>4 n-1, \bar{X}_{k+1}=A_{D} \bar{X}_{k}$, yielding $\operatorname{rank}\left(\bar{X}_{k+1}\right)=\operatorname{rank}\left(\bar{X}_{k}\right)$ since $A_{D}$ is non-singular. To conclude, $\bar{Y}_{k}=O_{D} \bar{X}_{k}$ where $O_{D}$ is the observability matrix which under the assumption $T \neq T_{i} / 2=\pi T_{i} / \omega_{i}$ is full rank proving item $i i$ ). Item $i$ ) directly follows from the previous case considering the reachability and observability properties inherited by subspaces of (2). Item $i i i)$ trivially holds as long as $T<\pi / \max _{i}\left\{\omega_{i}\right\}$.

It appears then evident that the results of Lemma 1 may be exploited to determine the correct number $n$ of frequencies and a suitable choice of the sampling time $T$. This is achieved by considering, for increasing values of the number of estimated frequencies $\hat{n}$, several (re-)samplings of measurements data on a moving window, associated to different values of the sampling time $T$, namely $T=p T_{s}$ with increasing $p=1,2, \ldots \bar{p}$, where $\bar{p}$ is the largest integer such that $\bar{p} T_{s}<\pi / \bar{\omega}$.

Then we evaluate the minimum eigenvalue $\lambda_{\min }\left(\bar{Y}_{k}^{\prime} \bar{Y}_{k}\right)$ and, more precisely, we highlight its dependency on $\hat{n}$ and $T$ by defining the function

$$
\Gamma(T, \hat{n}):=\lambda_{\min }\left(\bar{Y}_{k}^{\prime} \bar{Y}_{k}\right)^{-1}
$$

where $\bar{Y}_{k} \in \mathbb{R}^{2 \hat{n} \times 2 \hat{n}}$ and (see (3)) $Y_{k}=\left[y\left(t_{k}-2 \hat{n} T\right), \ldots, y\left(t_{k}-T\right)\right]^{\prime}$. According to the implication discussed in item $\mathrm{iii}$ ) of Lemma 1 , the correct value of the number of the estimated frequencies is equal to $\hat{n}=\bar{n}-1$, where $\bar{n}$ is the smallest integer such that the function $\Gamma(\cdot, \bar{n})$ takes arbitrarily large values for all (quantized) sampling times $T$ in the range between $T_{s}$ and $\bar{p} T_{s}$. Once the value $\bar{n}$ has been computed, a desirable selection for the sampling time is equal to $\bar{T}=\arg \min _{T}\{\Gamma(T, \bar{n}-$ $1)\}$. This choice is motivated directly by the proof of Theorem 1 that relies on the positivedefiniteness of the matrix $\bar{Y}_{k}^{\prime} \bar{Y}_{k}$, i.e. on the existence of a constant $\delta>0$ such that

$$
\bar{Y}_{k}^{\prime} \bar{Y}_{k}>\delta I
$$

with $\delta=\lambda_{\min }\left(\bar{Y}_{k}^{\prime} \bar{Y}_{k}\right)$. Note that this selection increases the convergence time of the "slowest" converging error $e_{i}=\omega_{i}-\hat{\omega}_{i}$. Nevertheless there might be other values of $T$ leading to faster transients. 


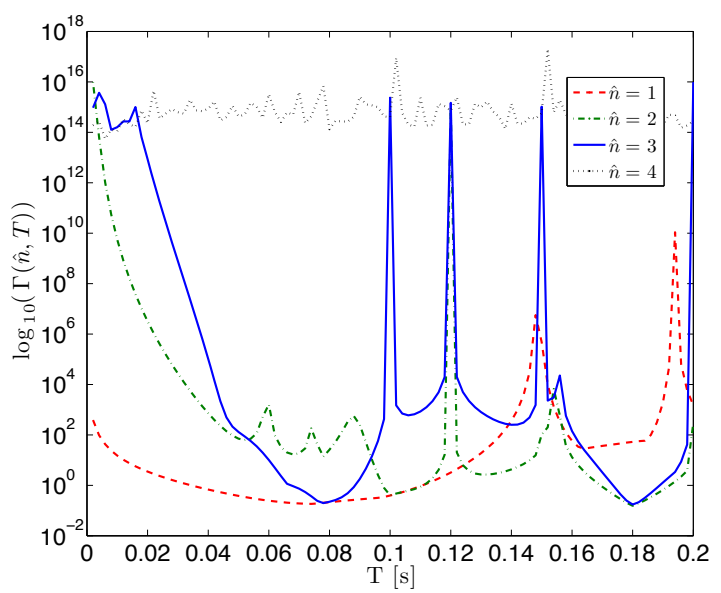

Figure 1. Dependence of the condition number of $\bar{Y}_{k}$ as a function of the sampling time $T$ and $\hat{n}$ with the measured signal (30) and $n=3$.

\section{Remark 4}

To determine the correct $n$, it is also possible to invert the matrix $\bar{Y}_{k}$ and obtain $\hat{a}$ using (10) for different $n$, selecting the one which minimizes $\left|y\left(t_{k}\right)-\hat{a} Y_{k}\right|$. However, numerically determining the minimum eigenvalue of $\bar{Y}_{k}^{\prime} \bar{Y}_{k}$ is typically more computationally efficient.

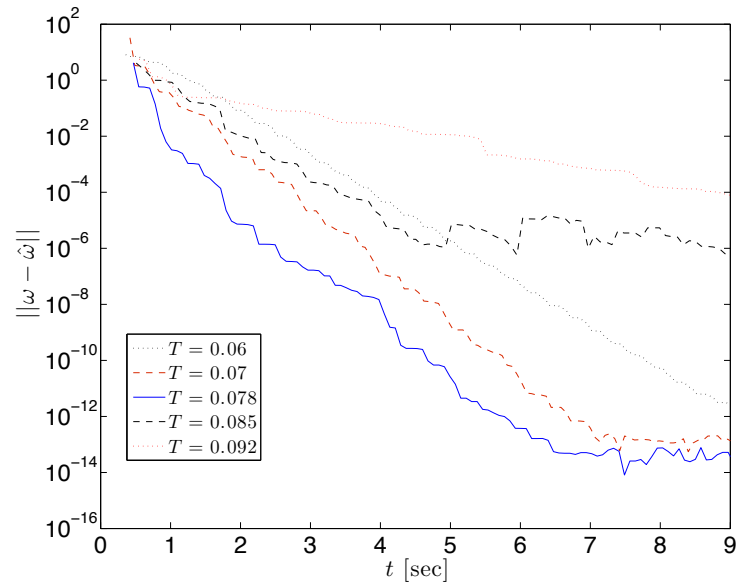

Figure 2. Simulation result: convergence of the estimation error with different sampling times $T$.

An illustration of the function $\Gamma(T, \hat{n})$ is shown in Fig. 1 in the presence of a signal (1) described by

$$
y(t)=\sin (t 2 \pi / 0.7)+\sin (t 2 \pi / 0.3)+\sin (t 2 \pi / 0.2)
$$


and $T_{s}=0.002$. It is evident that for $\hat{n}=n=3$ the first peak corresponds to 0.1 that is half of the shortest period 0.2 among the sinusoids composing $y(t)$, namely $0.1=\pi / \max _{i}\left\{\omega_{i}\right\}$. If $\hat{n}>n$ the function takes arbitrarily large values, denoting $\operatorname{rank}\left(\bar{Y}_{k}\right)<2 \hat{n}$. Note as well the large values of $\Gamma(T, \hat{n})$ for very small $T$. This phenomenon is due to the fact that the variation of the signal is negligible among different samples and the columns of $\bar{Y}_{k}$ are "numerically" dependent (badly conditioned). In the example of the signal (30), once $\hat{n}=3$ has been selected, the optimal $T$ is equal to $\arg \min _{T}\{\Gamma(T, \hat{n})\}=0.078$. The performance of the estimation provided by $\mathcal{H}$ using (14b)-(14c) with (28) and (29) and

$$
e=y\left(t_{k}\right)-Y_{k}^{\prime} \hat{S}\left[\begin{array}{c}
1 \\
f(\hat{\omega})
\end{array}\right], \hat{S}=\left[\begin{array}{cccc}
1 & 0 & 0 & 0 \\
0 & 1 & 0 & 0 \\
0 & 0 & 1 & 0 \\
0 & 0 & 0 & 1 \\
0 & 0 & 1 & 0 \\
0 & 1 & 0 & 0
\end{array}\right]
$$

is shown in Fig. 2, where the norm of the estimation error $\|\omega-\hat{\omega}\|$ for different values of $T$ are depicted.

We also show in Fig. 3 the time evolution of the estimates with $T=0.078$ which minimizes the function $\Gamma(T, \hat{n})$ selecting $\hat{n}=3, \gamma=1$, and $\hat{\omega}_{i}(0,0)=i$ for $i=1,2,3$. For numerical reasons we have selected $\gamma=1$ and let $\mathcal{H}$ flow for an equivalent time of 10 s among each sampling time $T$. The same result can be obtained integrating the flow map for $T$ seconds, i.e. within the inter-sample time, with $\gamma=10 / T$. However, to improve numerical integration it is more convenient to select a smaller $\gamma$ and a longer "virtual" flowing time between jumps. This can be done on-line accordingly to the computational capability of the hardware implementing $\mathcal{H}$.

In the presence of noise it is possible to render the algorithm robust to such noises by considering additional measurements, namely by increasing the length of $Y_{k}$. An example of the function $\Gamma(T, \hat{n})$ when the signal (30) is affected by additive uniformly distributed random noise between $[-0.2,0.2]$ (equivalently to $20 \%$ of the sinusoidal components amplitude) is shown in Fig. 4, where $\Gamma(T, \hat{n})$ is evaluated using $\nu$ additional samples that are stacked at the bottom of $Y_{k} \in \mathbb{R}^{(2 n+\nu) \times 2 n}$. Note that the difference of $\Gamma(T, \hat{n})$ between the correct value $\hat{n}=3=n$ and $\hat{n}=4>n$ is smaller than in Fig. 1.

\subsection{Self-tuning algorithm}

In the previous section, we have discussed how $\hat{n}$ and $T$ may be suitably selected by analyzing a window of the past measurements by means of the function $\Gamma$. We propose now a numerical technique to detect the correct value of the number of frequencies introducing a $N$-dimensional vector $\mathcal{I}(k)$, where $N$ is the largest possible number of frequencies, the $i$-th component of which 


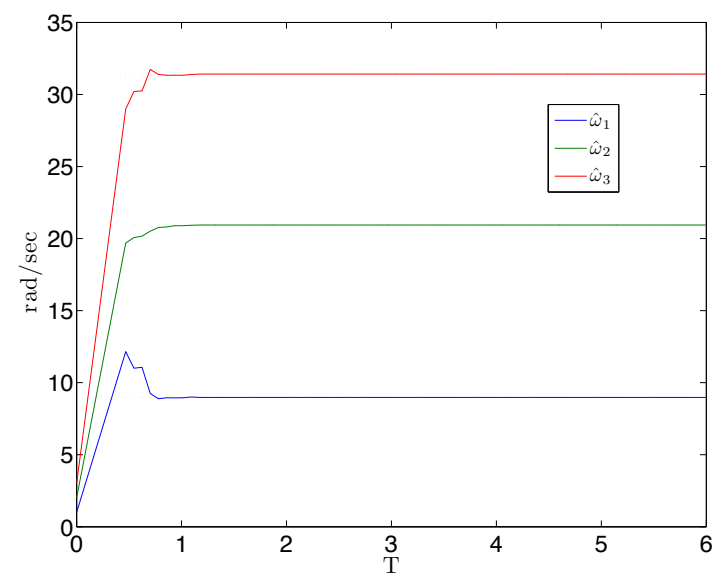

Figure 3. Estimated angular frequencies when $\hat{n}=n, T=0.078, \gamma=1$, and $\hat{\omega}_{i}(0,0)=i$ for $i=1,2,3$.

takes values in $\{0,1,2\}$, for different $p, T=p T_{s}$, as follows

$$
\mathcal{I}_{i}(p)=\left\{\begin{array}{lll}
0 & \text { if } \quad & \mathcal{I}_{i}(p-1)=0 \wedge g_{i}(p)>S_{a} G_{s, i} \\
1 & \text { if } \quad & \left(\mathcal{I}(p-1)=1 \wedge g_{i}(p)<S_{b} G_{s, i}\right) \vee \\
& \left(\mathcal{I}_{i}(p-1)=0 \wedge g_{i}(p)<S_{a} G_{s, i}\right) \\
& \text { if } \quad\left(\left(\mathcal{I}(p-1)=1 \wedge g_{i}(p) \geq S_{b} G_{s, i}\right) \wedge\right. \\
& & \left.\mathcal{I}_{i+1, \ldots, N}(p)<1\right) \vee \mathcal{I}_{i}(p-1)=2
\end{array}\right.
$$

where $g_{i}(p)=\Gamma\left(p T_{s}, i\right), G_{s, i}=\Gamma\left(T_{s}, i\right), S_{b}>S_{a}>0$ are threshold values. The jump map of the variable $\mathcal{I}_{i}$ is such that whenever $\mathcal{I}_{i}=2$ then $n=i$. In fact if $i<n$, the function $\Gamma\left(p T_{s}, i\right)$ decreases as $p$ grows to reach a minimum and then it increases as $p T_{s}$ approaches $\pi / \omega_{\max }$.

The threshold value $S_{a}$ and $S_{b}$ have been added to detect that the first (possibly local) minimum has been reached. An example of the relay-like graph of $\mathcal{I}_{i}$ is shown in Fig. 5. Assuming that a data buffer $y_{b}$ of the past input $y$ is available to construct $\bar{Y}_{k}$ for different values of the sampling time, the algorithm to select $\hat{n}$ and $T$ is the following.

\section{Algorithm 1}

1) Select $N, S_{a}, S_{b}$. Set $\hat{n}=0, p=2, \mathcal{I}_{i}(1)=0$ and evaluate $G_{s, i}=\Gamma\left(T_{s}, i\right)$ for $i=1, \ldots, N$.

2) While $\max _{i=1, \ldots, n} \mathcal{I}_{i}(p)<2$ do

Evaluate $\mathcal{I}_{i}(p)$ by (31) with $T=p T_{s}$ and for $i=1, \ldots, N$. Set $p=p+1$.

3) $\hat{n}=\operatorname{argmax}_{i=1, \ldots, N} \mathcal{I}_{i}, T=\operatorname{argmin}_{\tau} \Gamma(\tau, \hat{n})=p T_{s}$.

\section{Remark 5}

The algorithm can be readily extended to the case where $N$ dynamically grows and the a priori bound $n<N$ is not known. 


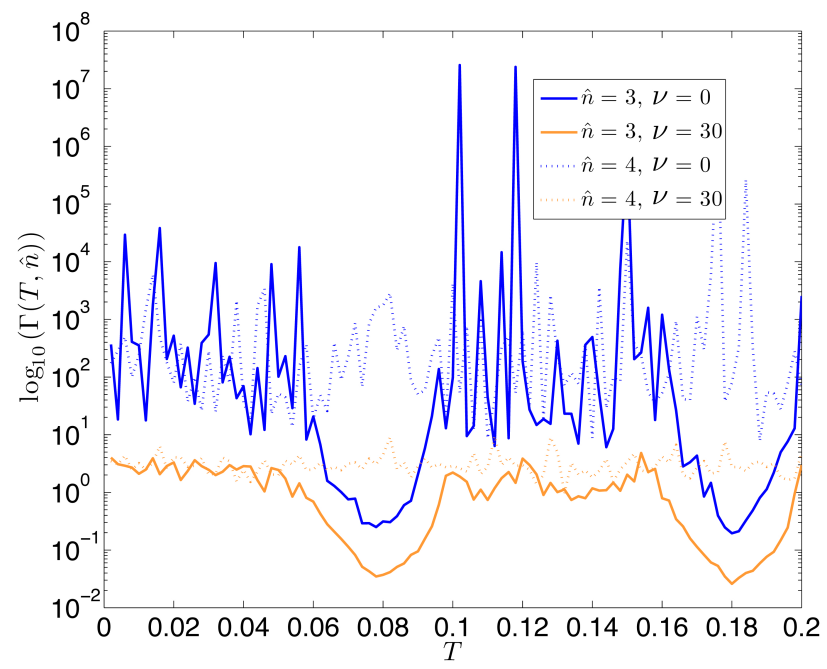

Figure 4. The function $\Gamma(T, \hat{n})$ in presence of additive noise on the signal (30) considering extra samples $(\nu=30)$.

To continuously evaluate on-line $\hat{n}$ and $T$, we propose an algorithm to verify if $\omega$ or $n$ have changed defined as follows.

\section{Algorithm 2}

For each new sample:

\section{if $\hat{n}=0$ : run Algorithm 1}

else : if $|e(t, k)|=\left|y\left(t_{k}\right)+Y_{k}^{\prime} \hat{S}\left[1, \hat{a}_{c}(t, k)^{\prime}\right]^{\prime}\right|>S_{e}|| y\left(t_{k}\right)||$ set $\hat{n}=0$ and empty the data buffer $y_{b}$.

The parameters that have to be selected are $S_{a}>0, S_{b}>0$ such that $S_{a}<S_{b}$, and $S_{e}>0$. An example is discussed in Section 5. Note that $S_{e}$ limits the number of time the Algorithm 1 is performed.

\section{Theorem 2}

Let Assumption 1 hold and assume that $\omega(t)$ and $n(t)$ are piecewise constant. Perform the Algorithm 2 to determine $\hat{n}$ and $T$ at each new sample of $y$ and, whenever $\hat{n}(t)>0$, evaluate the dynamics (14) of $\mathcal{H}$. Let $\tilde{a}_{c}(t, k):=a_{c}-a(t, k)$, then provided $\omega(t)$ and $n(t)$ vary sufficiently slow such that $\hat{n}(t)=n, t \in\left[t_{k}, t_{k+1}\right]$, it holds

$$
\left\|\tilde{a}_{c}(t, k)\right\| \leq\left\|\tilde{a}_{c}\left(t_{\bar{k}}, \bar{k}\right)\right\| e^{\sigma(t)\left(t-t_{\bar{k}}-4 n T\left(t_{k}\right)\right)},
$$

with $\sigma(t):=\lambda_{\min }\left(\bar{Y}_{k}^{\prime} \bar{Y}_{k}\right)$, and

$$
Y_{k}:=\left[y\left(t_{k}-2 n T\left(t_{k}\right)\right), \ldots, y\left(t_{k}-T\left(t_{k}\right)\right), y\left(t_{k}\right)\right]^{\prime},
$$

otherwise if $\hat{n}(t)>0$, then

$$
|e(t, k)|=\left|y\left(t_{k}\right)+Y_{k}^{\prime} \hat{S}\left[1, \hat{a}_{c}(t, k)^{\prime}\right]^{\prime}\right| \leq S_{e}|| Y_{k} \| .
$$




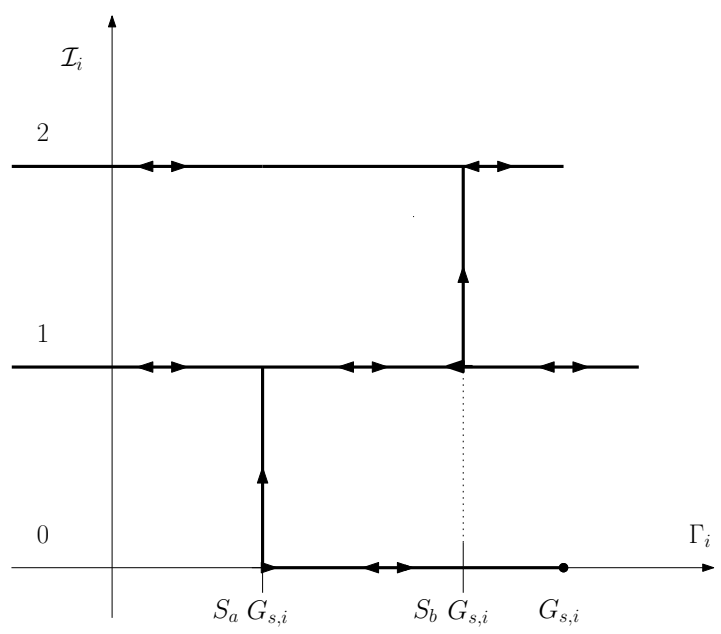

Figure 5. The graph of $\mathcal{I}_{i}$.

Proof: Note that if $\omega(t)$ and $n(t)$ do not change long enough such that $\hat{n}(t)=n, t \in\left[t_{k}, t_{k+1}\right]$, and given that the Algorithm 2 requires a time equal or less than $4 n T\left(t_{k}\right)$ to find the true value $\hat{n}=n$, then (32) holds since the exponential convergence to zero of the estimation error is inherited directly from Theorem 1 . In all the other case that an estimate of $n$ is found but it is not correct, namely $n \neq \hat{n}>0$, then (see the Algorithm 2 ) $\hat{n}$ is not reset to zero only if the inequality (33) is satisfied.

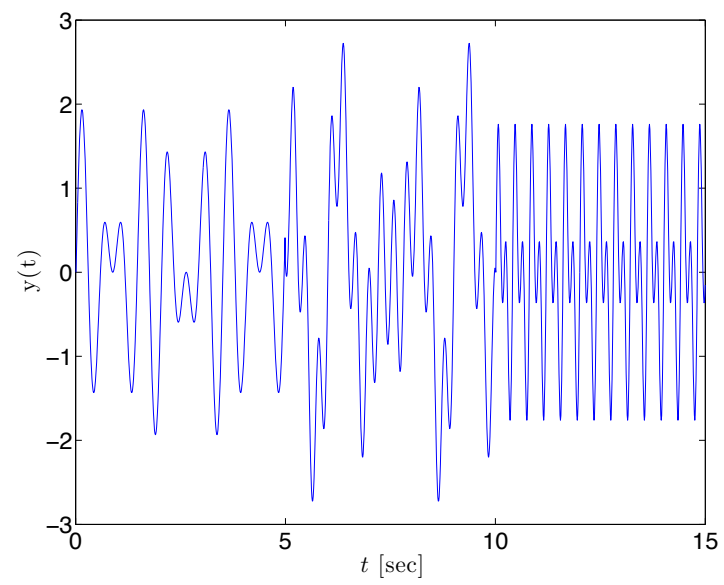

Figure 6. The signal $y(t)$ with changing frequencies. 
We show the effectiveness of the observer to determine the exact number of frequencies with the signal (see Fig. 6)

$$
y(t)=\left\{\begin{array}{cl}
\sin (2 \pi / 0.7 t)+\sin (2 \pi / 0.5 t) & t \in[0,5) \\
\sin (2 \pi / 1.5 t)+\sin (2 \pi t)+\sin (2 \pi / 0.3 t) & t \in[5,10) \\
\sin (2 \pi / 0.4 t)+\sin (2 \pi / 0.2 t) & t \geq 10 .
\end{array}\right.
$$

The observer thresholds have been selected equal to $\left[S_{a}, S_{b}, S_{e}\right]=\left[0.7,0.8,10^{-4}\right]$. Note that the selection of these parameters do not depend on the amplitude and the frequency of the sinusoidal terms in (34), but from the signal/noise ratio.

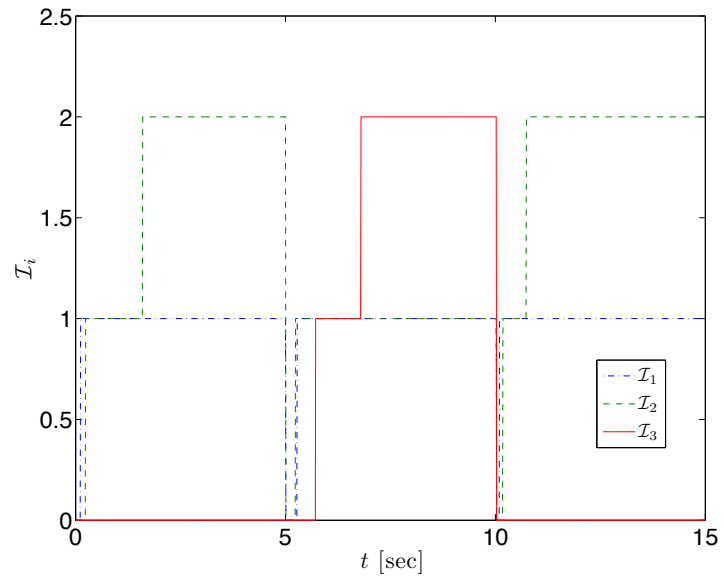

Figure 7. The graph of $\mathcal{I}_{i}$ with the signal (34).

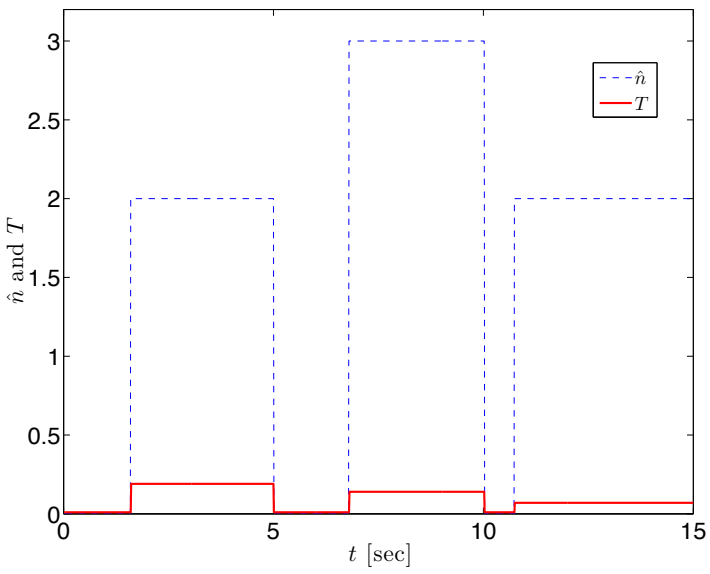

Figure 8. The estimated number of frequencies and the associated $T$ with the signal (34). 
Additional simulations have also been considered with additive random noise uniformly distributed between $[-0.3,0.3]$. The graphs reporting the time histories of the number of estimated frequencies and of the sampling time $T$ are not shown since they are identical to those in Fig. 8. To achieve this result, the observer thresholds have been selected as $\left[S_{a}, S_{b}, S_{e}\right]=[0.3,0.8,0.8]$ and the matrix $Y_{k} \in \mathbb{R}^{2 n+40}$ has been enlarged to contain 40 extra samples. Note that $S_{e}$ (see the Algorithm 2) needs to be increased in the presence of measurement noise.

\section{ROBUST REGULATOR DESIGN}

In this section we exploit the hybrid observer for multi-frequency signals designed above to tackle the challenging problem of output regulation for linear systems in which the exosystem is not known a priori.

\subsection{Problem definition and preliminaries}

Consider a linear plant $\mathcal{P}$ described by the equations

$$
\begin{aligned}
& \dot{x}=A x+B u+P w, \\
& e=C x+D u+Q w,
\end{aligned}
$$

with state $x \in \mathbb{R}^{n_{x}}$, control input $u \in \mathbb{R}^{p}$ and performance output $e \in \mathbb{R}^{q}$. The exogenous signal $w \in \mathbb{R}^{m}$, which may contain both reference signals to be tracked as well as disturbances to be rejected, is assumed to be generated by the following exosystem $\mathcal{S}$ :

$$
\dot{w}=A_{\omega} w,
$$

with $A_{\omega}$ defined as in the signal generator (2). According to the framework considered herein, the matrix $A_{\omega}$ as well as the interconnection matrices $P$ and $Q$ are assumed to be unknown, apart from the structure of $A_{\omega}$. Note that the $i$-th row $P_{i}$ selects the components (frequencies) of $w$ affecting the state $x_{i}$. As an example, if $P_{i}=C_{\omega}$, then $P_{i} w=y$ as defined in (1).

Let $P_{\Sigma}(s)$ denote the system matrix of system (35), that is,

$$
P_{\Sigma}(s):=\left[\begin{array}{cc}
A-s I & B \\
C & D
\end{array}\right] .
$$

The values $\bar{s} \in \mathbb{C}$ for which the complex-valued matrix $P_{\Sigma}(\bar{s})$ has rank less than the rank of $P_{\Sigma}(s)$ as a polynomial matrix constitute the invariant zeros or system zeros, which include all the transmission zeros as well as a subset of the input decoupling zeros (eigenvalues of the unreachable subsystem) and the output decoupling zeros (eigenvalues of the unobservable subsystem). The following assumption defines the class of plant models considered in this paper.

\section{Assumption 2}

In (35), the pair $(A, B)$ is stabilizable and the pair $(A, C)$ is detectable. 
The following assumption is a standard sufficient condition [3] for the solvability of the regulator problem (which becomes necessary if mild assumptions on parametric uncertainties affecting the plant matrices are considered). For a square matrix $A, \Lambda(A)$ denotes the set of the eigenvalues of $A$.

\section{Assumption 3}

$\operatorname{rank} P_{\Sigma}(\lambda)=n_{x}+q$, for all $\lambda \in \Lambda\left(A_{\omega}\right)$. This implies that no invariant zero of $(A, B, C, D)$ coincides with an eigenvalue of $A_{\omega}$

\section{Remark 6}

In the setting defined above we assume that both the reference signals and the disturbances consist of combination of sinusoids at different (unknown) frequencies. It may be also possible to assume that $w$ is partitioned as $w=\left[\begin{array}{ll}w_{r}^{\prime}, & w_{d}^{\prime}\end{array}\right]^{\prime}$, namely explicitly distinguishing between references $w_{r}$ and disturbances $w_{d}$, and that the components of the reference signals (to be tracked) are produced by perfectly known generators, i.e. $\dot{w}_{r}=A_{r} w_{r}$. The results of this section can be then easily extended to the case in which the references include, in the (known) matrix $A_{r}$, signals richer than combination of sinusoids, e.g. constant and polynomial signals to mention just a few.

The standard regulator (or servomechanism) problem can be stated as follows.

\section{Definition 1}

Given plant (35) and exosystem (36), find, if possible, a compensator that suitably connected to the plant ensures that

(i) the closed loop is asymptotically stable;

(ii) $\lim _{t \rightarrow+\infty} e(t)=0$, for any initial state of the exosystem (36), of the plant (35) and of the compensator.

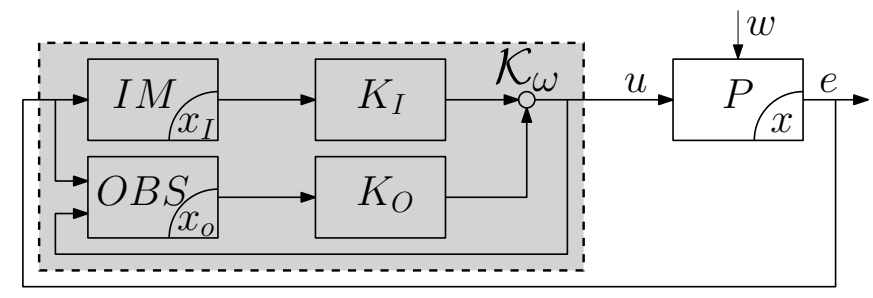

Figure 9. A regulator solving the standard problem

\subsection{Servocompensator design}

A solution to the above problem is given by a compensator $\mathcal{K}_{\omega}$ consisting of three main components (see Fig. 9):

- an internal model of the exogenous signals

$$
\dot{x}_{I}=A_{I} x_{I}+B_{I} e
$$

which is driven by the error signal (35b), with $\left(A_{I}, B_{I}\right)$ reachable, $A_{I}$ having the same eigenvalues of $A_{\omega}$, i.e. containing the same frequencies; 
- an observer for the undisturbed ( $w \equiv 0)$ plant (35a), namely

$$
\dot{x}_{o}=A x_{o}+B u+L\left(C x_{o}+D u-e\right)
$$

where $L$ is selected such that $(A+L C)$ Hurwitz;

- a stabilizing state feedback

$$
u=K\left[\begin{array}{l}
x_{I} \\
x_{o}
\end{array}\right]=\left[\begin{array}{ll}
K_{I} & K_{o}
\end{array}\right]\left[\begin{array}{l}
x_{I} \\
x_{o}
\end{array}\right]
$$

for the cascade interconnection of the plant (with $w \equiv 0$ ) and the internal model

$$
\left[\begin{array}{c}
\dot{x}_{I} \\
\dot{x}
\end{array}\right]=\left[\begin{array}{cc}
A_{I} & B_{I} C \\
0 & A
\end{array}\right]\left[\begin{array}{c}
x_{I} \\
x
\end{array}\right]+\left[\begin{array}{c}
B_{I} D \\
B
\end{array}\right] u .
$$

The three components are combined together in the compensator

$$
\begin{aligned}
& \dot{x}_{c}=A_{c} x_{c}+B_{c} u_{c}, \\
& y_{c}=C_{c} x_{c}+D_{c} u_{c},
\end{aligned}
$$

where $x_{c}=\left[\begin{array}{ll}x_{I}^{\prime} & x_{o}^{\prime}\end{array}\right]^{\prime}$ and

$$
\begin{aligned}
& A_{c}=\left[\begin{array}{cc}
A_{I} & 0 \\
(B+L D) K_{I} & (A+L C)+(B+L D) K_{o}
\end{array}\right], \\
& B_{c}=\left[\begin{array}{c}
B_{I} \\
-L
\end{array}\right], \quad C_{c}=\left[\begin{array}{ll}
K_{I} & K_{o}
\end{array}\right], \quad D_{c}=0,
\end{aligned}
$$

to be connected to the plant according to the structure

$$
u_{c}=e, \quad u=y_{c} .
$$

For later use, the dependence of compensator $\mathcal{K}_{\omega}$ on $\omega$ is made explicit in the notation, where $\omega$ is the vector containing the frequencies of the signals generated by (36). The following classical result asserts the effectiveness of compensator (38), (39) in solving the problem in Definition 1.

\section{Theorem 3}

If Assumption 2 and Assumption 3 hold, then the problem in Definition 1 is solvable and a solution is given by compensator (38), (39) with the interconnection (40).

As anticipated above, the focus of this paper is on the case when the exosystem in (36) is unknown, i.e. the number $m=2 n<N$ and the value of the frequencies $\omega$, with $\left|\omega_{i}-\omega_{j}\right|>\varepsilon$ for all $(i, j) \in\{1, \ldots, n\}$, are unknown. The problem can be stated as follows.

\section{Definition 2}

Given a plant (35) with known $(A, B, C, D)$ and an exosystem (36) with unknown $\left(A_{\omega}, P, Q\right)$, find, if possible, a compensator that suitably connected to the plant ensures that 
(i) the closed loop is asymptotically stable;

(ii) $\lim _{t \rightarrow+\infty} e(t)=0$, for any initial state of the exosystem (36), of the plant (35) and of the compensator.

Since $A_{\omega}$ is unknown in the problem Definition 2, it is expected that Assumption 3 (which is a necessary condition for the solvability of the problem in Definition 1 under mild assumptions on the errors affecting the nominal values of the parameters of $A$ ) must be strengthened in order to guarantee that the required property holds for any admissible $A_{\omega}$. For this reason, Assumption 3 is replaced by the following Assumption 4.

\section{Assumption 4}

$\operatorname{rank} P_{\Sigma}(\jmath \omega)=n_{x}+q, \forall \omega \in \mathbb{R}$.

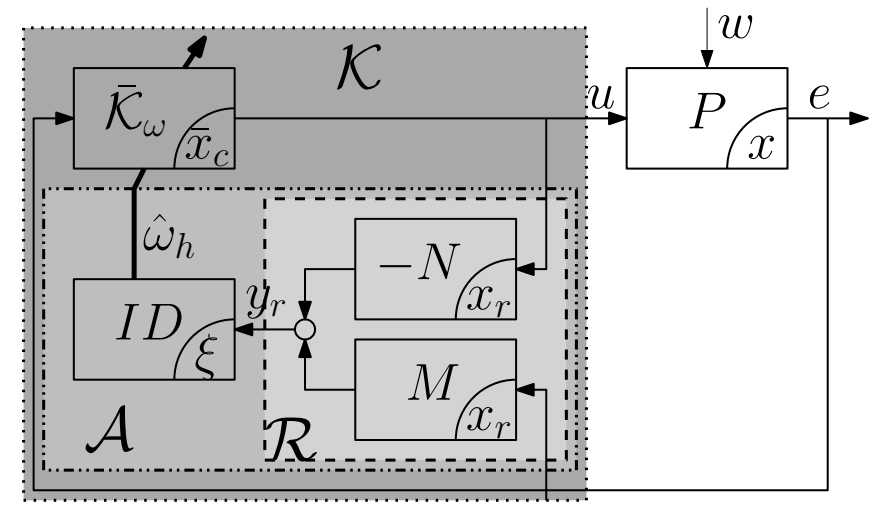

Figure 10. The adaptive hybrid robust regulator solving the adaptive problem (note that $N, M$ share the same state, i.e. are represented by two blocks but are implemented by a single system (41)).

\section{ARCHITECTURE OF THE HYBRID ADAPTIVE REGULATOR}

The proposed controller $\mathcal{K}$ consists of three main components (see Fig. 10):

- a residual generator $\mathcal{R}$, which provides a signal suitable for the identification of the frequencies contained in the exogenous reference/disturbance;

- the frequency identifier ID, which produces an estimate $\omega$ of the frequencies present in $w$;

- the switching servocompensator $\overline{\mathcal{K}}_{\omega}$, which provides an internal model based on the estimated frequencies $\omega$ together with stabilizing control action.

The three components are separately discussed in the following sections (the second one has been extensively dealt with in Section 2 and it is here simply adapted to the regulation framework).

\subsection{The residual generator $\mathcal{R}$}

Towards the definition of such generator let $L_{r}$ be such that $\left(A+L_{r} C\right)$ is Hurwitz. This selection is always possible by Assumption 2. The residual generator is a LTI continuous-time system described 
by the equations

$$
\begin{aligned}
\dot{x}_{r} & =\left(A+L_{r} C\right) x_{r}-\left(B+L_{r} D\right) u+L_{r} e, \\
y_{r} & =C x_{r}-D u+e
\end{aligned}
$$

In the Laplace domain system (41) yields a (matrix) transfer function given by $\left[\begin{array}{ll}-N(s) & M(s)\end{array}\right]$ where $N(s) \in \mathcal{R H}_{\infty}$ and $M(s) \in \mathcal{R} \mathcal{H}_{\infty}$ provide a right coprime factorization of the matrix transfer function of (35) from $u$ to $e$, namely [31]

$$
M^{-1}(s) N(s)=C(s I-A)^{-1} B+D .
$$

Note that, if the plant is (open-loop) stable, the factorization (42) can be simply replaced by $M(s)=I, N(s)=C(s I-A)^{-1} B+D$.

\section{Remark 7}

The main role of (41) is to guarantee that the frequency identifier is virtually in open loop, in the sense that $y_{r}$ is independent from $u$ in Fig. 10 (the corresponding transfer function is zero, as shown below). In fact, consider a complete right coprime factorization of (35) such that (42) is complemented with

$$
M^{-1}(s) N_{w}(s)=C(s I-A)^{-1} P+Q
$$

consider also zero initial conditions (otherwise, an additional exponentially converging term would also appear, which bears no consequence on the closed-loop stability analysis). It is then possible to write

$$
\begin{aligned}
y_{r}(s) & =M(s) e(s)-N(s) u(s) \\
& =M(s) M^{-1}(s)\left[N(s) \quad N_{w}(s)\right]\left[\begin{array}{l}
u(s) \\
w(s)
\end{array}\right]-N(s) u(s) \\
& =N_{w}(s) w(s),
\end{aligned}
$$

As a consequence, the combination of the plant, the residual generator and the frequency identifier (from $u$ to the output of the frequency identifier) will essentially behave as an open loop stable filter on $w$, and if switching stability of the closed loop system in Fig. 10 without $\mathcal{A}$ is achieved, then stability of the overall adaptive closed loop system in Fig. 10 will also follow. A small gain reasoning can be used to show that the same conclusions still hold in the presence of sufficiently small mismatch between the real plant and the model (35).

\section{Remark 8}

One could be tempted to use only the error signal $e$ instead of $y_{r}$ for frequency detection. In order to see why this choice is not advisable, consider the case of an exogenous signal composed by a single sinusoid at frequency $\omega_{1}$ for $t \in\left[t_{0}, t_{1}\right)$, to which a second sinusoid at frequency $\omega_{2}$ is added for $t \in\left[t_{1},+\infty\right)$. Assume also that at time $\tau_{1} \in\left[t_{0}, t_{1}\right)$ the first sinusoid has been identified, and almost completely compensated in the error signal $e$. After time $t_{1}$, the frequency identifier would start detecting the sinuosoid at frequency $\omega_{2}$ (but not the one at frequency $\omega_{1}$, which is already compensated for) and then at the next controller switch at time $\tau_{2}$ the internal model would be 
designed to compensate for the sinusoid at frequency $\omega_{2}$ but not for the one at frequency $\omega_{1}$. The same process would then happen again with the roles of $\omega_{1}$ and $\omega_{2}$ exchanged, thus leading to cycles without convergence of $e$ to zero. It is easy to see that this kind of problems does not arise when the control input information is suitably elaborated, as is done by (41). The dynamics of (41) can also be used to introduce a suitable filtering action on high frequency noise possibly affecting the measurements of $e$.

\section{Remark 9}

To estimate $\omega$ without relying on the residual generator, which involves an exact cancellation, it is possible to consider $p+q$ adaptive observers that are fed with the components of the vectors $u_{f} \in \mathbb{R}^{p}$ and $e_{f} \in \mathbb{R}^{q}$, which are the output of MIMO filters capable of removing constant bias of $u$ and $e$. Standard high (band-)pass filters can be considered fur such purpose. The frequencies identified by each adaptive observer are then merged into the single frequency vector $\hat{\omega}_{h}$, avoiding the duplication of all the estimated frequencies of the signals $u_{f}$ and $e_{f}$ that differs less than $\varepsilon$. This approach, although $p+q$ adaptive observers have to be implemented, permits the construction of a regulator that is robust with respect to uncertainties in the plant in addition to robustness with respect to the exosystem.

\subsection{Frequency identifier ID}

The hybrid observer extensively discussed in Section 2 is considered to identify the angular frequencies $\omega_{i}$ 's of the signal $w$ in (35), i.e. the non-zero eigenvalues on the imaginary axes of the matrix $A_{\omega}(36)$. To this aim, we define

$$
y_{\omega}\left(t_{k}\right):=C_{r}\left(y_{r}\left(t_{k}\right)-y_{r}\left(t_{k-1}\right)\right)
$$

with $C_{r}=[1,1, \ldots, 1] \in \mathbb{R}^{q}$ such that $y_{\omega}$ is scalar and it contains all the frequencies of $w$. Once the correct value $2 n=m$ of eigenvalues related to the sinusoidal components is obtained, an estimate $\hat{\omega}$ is provided by (14) with initial conditions $\hat{\omega}_{i}(0), i=1, \ldots, n$.

The piecewise constant signal $\hat{\omega}_{h}(t)$ fed to the servocompensator is the output of a sample and hold device with input $\hat{\omega}(t)$ and sampling time $T_{r}:=-\ln (\beta) / \lambda_{\min }\left(\bar{Y}_{k}^{\prime} \bar{Y}_{k}\right)$, where $0<\beta<1$ is a design parameter corresponding to the desired shrinking factor of the estimation error between each sampling time $T_{r}$. With this choice, the smaller the $\beta$ the fewer the number of switches of the servocompensator. At each new sample of $y_{\omega}$ the algorithm of Section 2 checks if the inequality $\left|y_{\omega}\left(t_{k}\right)+\zeta^{\prime} \hat{S}\left[1, f(\hat{\omega})^{\prime}\right]^{\prime}\right|>S_{e}\|\zeta\|=S_{e}\left\|Y_{k}\right\|$ is satisfied. This is needed to identify a possible variation in the number or the values of the frequencies. If such changing is detected, the past data collected in $Y_{k}$ are discarded and the procedure of determining the number of frequencies and the sampling time $T$ is iterated. In fact, even in the presence of time-varying $m(t)$ and $\omega(t)$, Theorem 2 can be invoked to show that if $m(t)$ and $\omega(t)$ are constant for $t \in[\bar{t}, \rho]$, with $\bar{t}+4 m(\bar{t}) T<\rho$, then for $t \in(-(\bar{t}+4 m(\bar{t}) T), \rho)$ the estimated number of frequencies is correct $(2 \hat{n}=m)$ and

$$
\|\omega(\rho)-\hat{\omega}(\rho, k)\| \leq e^{-\sigma(\rho-\bar{t}-4 m(\bar{t}) T)}\|\omega(\bar{t})-\hat{\omega}(\bar{t}, \bar{k})\|,
$$

for some $\bar{k}$ and $k$ and $\sigma=\lambda_{\min }\left(\bar{Y}_{k}^{\prime} \bar{Y}_{k}\right)$ with the selected $T$. Hence, a similar exponential bound clearly holds for $\hat{\omega}_{h}(t)$ too. 


\subsection{The switching servocompensator $\overline{\mathcal{K}}_{\omega}$}

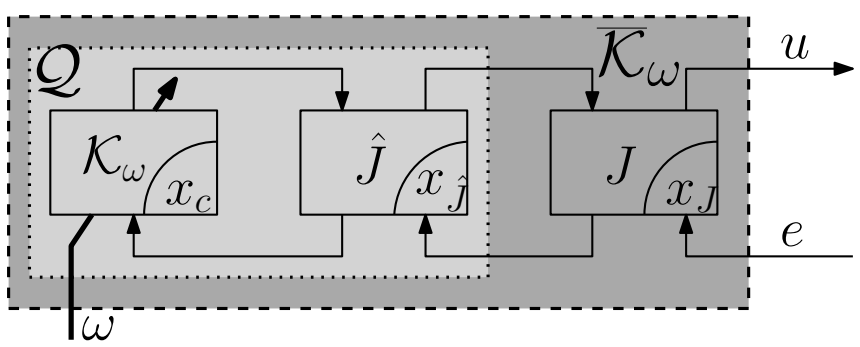

Figure 11. The Youla-Kucera based realization (45), (46) of the switching controller.

In order to ensure stability of the switching system, the switching servocompensator is implemented using a constant part (composed by the subsystem $J$ in Fig. 11) and a servocompensator $\mathcal{K}_{\omega}$ as in (38), (39) which is redesigned each time the signal $\hat{\omega}_{h}$ changes. By reasoning as in [32,33], if a suitable realization of this changing part is adopted, closed loop stability can be guaranteed for arbitrary switchings due to the changes in $\hat{\omega}_{h}$.

Denote by $\mathcal{F}_{L}(M, R)$ the lower fractional transformation

$$
\mathcal{F}_{L}(M, R):=M_{11}+M_{12} R\left(I-M_{22} R\right)^{-1} M_{21} .
$$

Let $K_{k}$ and $L_{k}$ be such that $A+L_{k} C$ and $A+B K_{k}$ are Hurwitz. The subsystems $J$ and $\hat{J}$ are characterized, respectively, by the matrices:

$$
\begin{aligned}
& {\left[\begin{array}{c|c}
A_{J} & B_{J} \\
\hline C_{J} & D_{J}
\end{array}\right]:=\left[\begin{array}{c|cc}
A+B K_{k}+L_{k} C+L_{k} D K_{k} & -L_{k} & B+L_{k} D \\
\hline K_{k} & 0 & I \\
-\left(C+D K_{k}\right) & I & -D
\end{array}\right],} \\
& {\left[\begin{array}{c|c}
A_{\hat{J}} & B_{\hat{J}} \\
\hline C_{\hat{J}} & D_{\hat{J}}
\end{array}\right]:=\left[\begin{array}{c|cc}
A & -L_{k} & B \\
\hline-K_{k} & 0 & I \\
C & I & D
\end{array}\right] .}
\end{aligned}
$$

By [31, Theorem 12.8], all stabilizing controllers for (35) are parameterized by the LFT

$$
K=\mathcal{F}_{L}(J, Q), Q \in \mathcal{R} \mathcal{H}_{\infty}, \operatorname{det}(I+D Q(\infty)) \neq 0
$$

conversely [31, Remark 12.9], the parameter $Q$ yielding the particular stabilizing controller $K$ can be expressed as

$$
Q=\mathcal{F}_{L}(\hat{J}, K)
$$

However, in general it is not enough to use $K=\mathcal{K}_{\omega}$ for the current value of $\hat{\omega}_{h}$ in order to achieve stability for any possible switching sequence; on the other hand, as shown in [32,33], such property holds if the realization of $Q$ in (46b) is suitably chosen. Although other approaches could be used to guarantee stability (taking into account, for example, that each updates of $\hat{\omega}_{h}$ requires a certain 
amount of time, and then a known dwell time is always guaranteed between two switchings $\|^{\|}$), this one is adopted here due to the ensuing simplified analysis (no additional care has to be taken to ensure stability).

For simplicity of implementation, it is useful to fix a maximum state space dimension for $Q$. Let $\bar{n}_{Q}:=n_{\text {exo }}+n_{x}$, where $n_{x}$ is state dimension of (35) and $n_{\text {exo }}$ is the maximum state dimension of the exosystem (36). When $\hat{\omega}_{h}$ changes, the following algorithm is performed:

- design a servocompensator $\mathcal{K}_{\omega}$ as in Section 3.2;

- find a minimal realization $\left(\hat{A}_{Q}, \hat{B}_{Q}, \hat{C}_{Q}, \hat{D}_{Q}\right)$ of $\hat{Q}:=\mathcal{F}_{L}\left(\hat{J}, \mathcal{K}_{\omega}\right)$ with $\hat{J}$ given in (45b);

- let $n_{Q}$ be the size of $\hat{A}_{Q}$ and define $\tilde{n}_{Q i}=\bar{n}_{Q}-n_{Q}$.

- let $T_{Q}=X^{\frac{1}{2}}$ where $X$ solves the Lyapunov equation:

$$
\hat{A}_{Q}^{T} X+X \hat{A}_{Q}=-I
$$

- given $\tilde{\alpha}>1$, define the realization of $Q$ as

$$
\left[\begin{array}{c:c:c}
A_{Q} & B_{Q} \\
\hdashline C_{Q} & D_{Q}
\end{array}\right]=\left[\begin{array}{cc:c}
T_{Q} \hat{A}_{Q} T_{Q}^{-1} & 0 & T_{Q} \hat{B}_{Q} \\
0 & -\tilde{\alpha} I_{\tilde{n}_{Q}} & 0 \\
\hdashline \hat{C}_{Q} T_{Q}^{-1} & 0 & 0
\end{array}\right],
$$

where the blocks having at least one dimension equal to $\tilde{n}_{Q}$ are absent if $\tilde{n}_{Q}=0$.

System $Q$ in Fig. 11 is then implemented as the switching system (where the dependence on $\omega$ of the state space matrices is highlighted)

$$
\begin{aligned}
& \dot{x}_{Q}=A_{Q(\omega)} x_{Q}+B_{Q(\omega)} u_{Q}, \\
& y_{Q}=C_{Q(\omega)} x_{Q}+D_{Q(\omega)} u_{Q},
\end{aligned}
$$

where $x_{Q}(\cdot): \mathbb{R}_{\geq 0} \mapsto \mathbb{R}^{\bar{n}_{Q}}$ has fixed dimension and is continuous at the switching times of $\hat{\omega}_{h}$, that is $x_{Q}(t)=x_{Q}\left(t^{-}\right)$for all $t$ even if $\omega(t) \neq \omega\left(t^{-}\right)$. With the above choices, all considered $Q$ 's have the same state dimension, and share $V\left(x_{Q}\right)=x_{Q}^{\prime} x_{Q}$ as a common Lyapunov function; moreover, such function satisfies $\dot{V}\left(x_{Q}\right) \leq-V\left(x_{Q}\right)$ when $u_{Q}=0$.

\section{Theorem 4}

Under Assumption 2 and Assumption 4, the hybrid compensator $\mathcal{K}$ solves the problem in Definition 2.

Proof: The closed loop system in Fig. 10 without $\mathcal{A}$ (hence considering $\hat{\omega}_{h}$ as an exogenous, piecewise constant signal taking values in a compact set) is exponentially stable, as can be proved by using the quadratic Lyapunov function $V\left(x, \bar{x}_{c}\right)=\bar{V}\left(x, x_{J}, x_{Q}\right)=V_{1}\left(x, x_{J}\right)+V_{2}\left(x_{Q}\right)$, where $V_{1}\left(x, x_{J}\right)$ is any quadratic Lyapunov function for the asymptotically stable linear time invariant system $\mathcal{F}_{U}(J, P)$, and $V_{2}\left(x_{Q}\right)=x_{Q}^{\prime} x_{Q}$ is a quadratic common Lyapunov function for all the dynamics that can be obtained for $Q$ by applying the algorithm in Section 4.3. By the reasoning in Remark 7, the output of the residual generator converges exponentially (due to possibly nonzero

"This hold true in our case $\hat{\omega}_{h}$ does not change before a minimum time equal to $4 m T_{s}$ elapses. 
initial conditions for the closed loop system) to the filtered signal having Laplace transform $N_{w}(s) w(s)$; in turn, due to the fact that $N_{w}(s)$ corresponds to a stable linear time invariant filter, the mentioned signal converges to a signal having the same frequency content of $w$. With such an input, the response $\hat{\omega}_{h}$ of the frequency identifier $I D$ converges exponentially to the true frequencies in $w$. From this and the properties of the classical regulator Section 3.2, it is easy to show that for any $w(0)$ and $\varepsilon>0$ there exists $T_{1}, T_{2}>0$ such that for $t>T_{1}$ the estimate $\hat{\omega}_{h}$ is sufficiently close to the real frequencies in $w$ to guarantee that the steady state error obtained using a regulator designed for the frequencies in $\omega$ yields a steady state error in response to $w$ smaller than $\varepsilon / 2$, so that for $t>T_{1}+T_{2}$ the actual error will be smaller than $\varepsilon$, that exponentially converges to zero as long as the matrix $A_{\omega}$ does not change.

\section{Remark 10}

If there exists at time $\bar{t}$ such that $m(t)$ and $\omega(t)$ are constant for $t \in[\bar{t}, \rho]$, with $\bar{t}+4 m(\bar{t}) T<\rho$, then for $t \in(-(\bar{t}+4 m(\bar{t}) T), \rho)$ it holds $\|e(t)\| \leq\|e(\bar{t})\| e^{-\sigma(\rho-\bar{t}-4 m(\bar{t}) T)}$ with $\sigma=\lambda_{\min }\left(\bar{Y}_{k}^{\prime} \bar{Y}_{k}\right)$ and $T$ selected by the $I D$.

\section{Remark 11}

By using reasonings similar to the proof of Theorem 4 and the additional robustness properties of the frequency identifier in Section 2, it is also possible to show that the proposed compensator can achieve practical (instead of asymptotic) regulation, in the sense that for any bound $\varepsilon>0$ on the norm of the acceptable error it is possible to find a bound $\delta>0$ such that if the measurement noise is smaller than $\delta$ than also the error will become smaller that $\varepsilon$ after a finite time. While the detailed description of such results is not provided here, it is mentioned here in order to substantiate the practical interest of the proposed method.

\section{NUMERICAL SIMULATIONS}

Consider the plant (35) with the matrices defined as

$$
\begin{aligned}
& A=\left[\begin{array}{cc}
-7.5 & 31 \\
-31 & 7.5
\end{array}\right], \quad B=\left[\begin{array}{c}
36 \\
68.5
\end{array}\right], \quad P=\left[\begin{array}{cc}
0 & 36 \\
0 & 68.5
\end{array}\right] \text {, } \\
& C=\left[\begin{array}{ll}
1 & 0
\end{array}\right], \quad D=0, \quad Q=\left[\begin{array}{ll}
-1 & 1
\end{array}\right],
\end{aligned}
$$

sampled with $T_{H}=0.02$, subject to the external multi-frequency signal $w(t)=$ $\left[\begin{array}{llll}\sin (2 t) & \cos (2 t) & 1.5 \sin (4 t) & 1.5 \cos (4 t)\end{array}\right]^{\prime}$. In particular the function $\sin (2 t)$ represents a reference signal whereas $1.5 \sin (4 t)$ describes a disturbance affecting the system. The initial conditions of the servocompensator are set to zero. The resulting tracking error and control input are shown in Fig. 12 and Fig. 13, respectively. Once the number of frequencies have been identified, which occurs approximatively after 7.3 seconds, it is possible to appreciate the fast convergence of the tracking error to zero. 


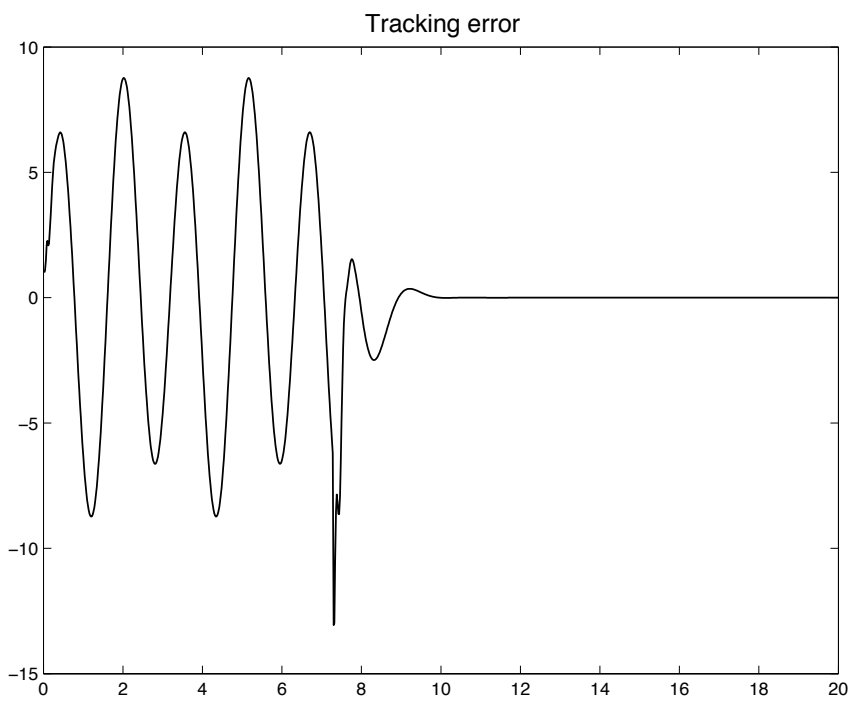

Figure 12. The tracking error in case of two frequencies.

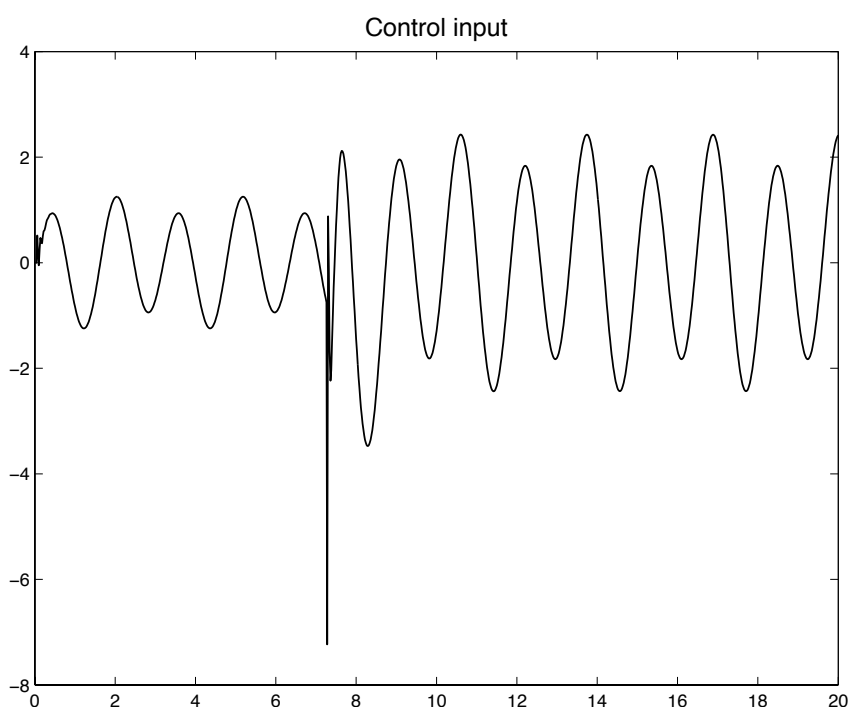

Figure 13. The control input in case of two frequencies

Consider again system (49), with $T_{s}=1 / 70$, in the presence time-varying $m(t)$ and $\omega(t)$ as

$$
\begin{aligned}
& m(t)=\left\{\begin{array}{lcc}
2 & \text { if } & t<100 / 7 \\
3 & \text { elsewhere }
\end{array}\right. \\
& \omega(t)=\left\{\begin{array}{cc}
(1,4) & \text { if } \\
(2,6,10) & \text { elsewhere }
\end{array}\right.
\end{aligned}
$$

suggesting a richer frequency content of the exogenous signal for $t \geq 100 / 7$. The estimated number of frequencies in this case is shown in Fig. 14 (note that the frequency estimator outputs a zero value each time the correct number of frequency of $y_{r}(t)$ is not retrieved). The tracking error and 
the control input are shown in Fig. 15 and Fig. 16, respectively. Note that two curves are shown in each figure, which correspond, respectively, to the case when the control input is saturated at the value 10 (blue curve) and to the case when the control input is not saturated (red curve). The rationale for limiting the control input is to reduce the transient that is triggered when the controller parameters are switched; in fact, although the state of the controller is continuous, the steady-state solutions corresponding to different values of $\omega$ are different, and such mismatch inevitably induces a transient response.

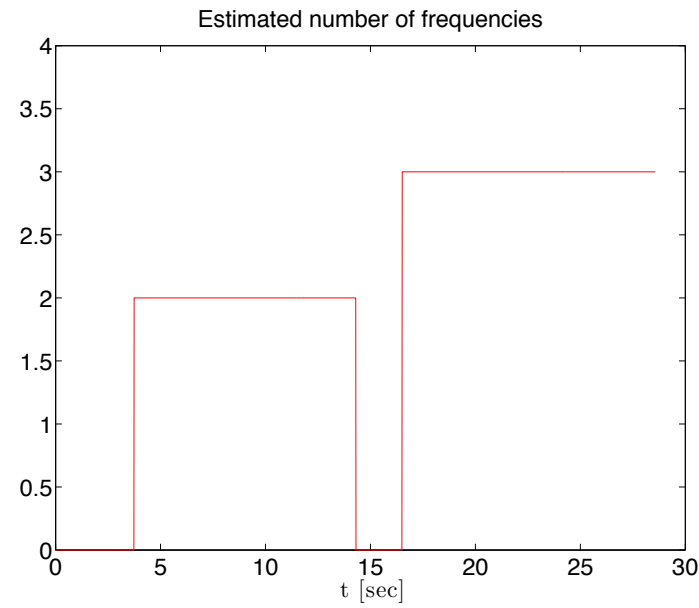

Figure 14. The number of frequency estimated by ID.

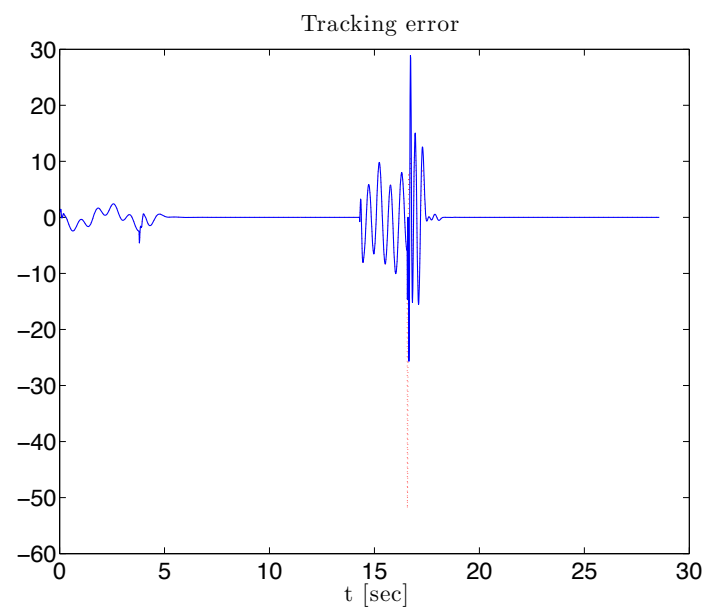

Figure 15. The tracking error in case of time varying frequencies with (solid) and without (dots) input saturation $(|u| \leq 10)$. 


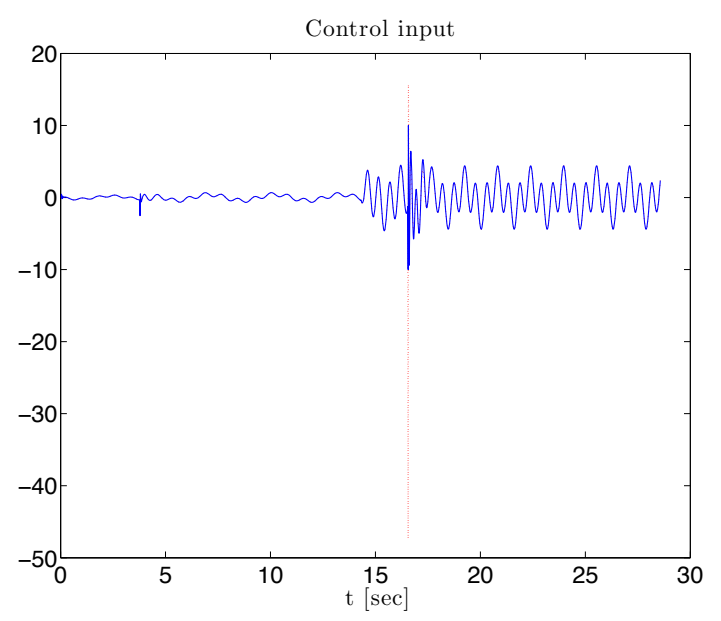

Figure 16. The control input in case of time varying frequencies with (solid) and without (dots) input saturation $(|u| \leq 10)$.

\section{CONCLUSIONS}

In this paper we have approach the problem of output regulation for LTI systems in the presence of unknown exosystems. The only a priori knowledge about the exosystem is that it generates a multi-frequency signal (unknown number of frequencies), with the single frequencies (as well as their amplitude and initial phase) being not known. The proposed solution hinges upon two main building blocks. To begin with, we have defined a hybrid observer to estimate the number and the values of the frequencies contained in the signal (1). The observer has been completed by an algorithm to autonomously adjust the number of estimated frequencies and a suitable (re-)sampling time $T$ to improve the frequency estimator performance. The proposed method has been tailored for numerical implementation. Then, based on the hybrid frequency estimator, the design of a novel regulator that is robust with respect plant parameters and perturbation in the exogenous signal is proposed .

\section{REFERENCES}

1. Francis B, Sebakhy O, Wonham W. Synthesis of multivariable regulators: The internal model principle. Applied Mathematics and Optimization 1974; 1(1):64-86, doi:10.1007/BF01449024. URL http://dx.doi.org/10.1007/BF01449024.

2. Wonham WM. Linear multivariable control- A geometric approach. Springer-Verlag, 1979.

3. Davison E. The robust control of a servomechanism problem for linear time-invariant multivariable systems. Automatic Control, IEEE Transactions on Feb 1976; 21(1):25-34, doi:10.1109/TAC.1976.1101137.

4. Davison E. Multivariable tuning regulators: The feedforward and robust control of a general servomechanism problem. Decision and Control including the 14th Symposium on Adaptive Processes, 1975 IEEE Conference on, 1975; 180-187, doi:10.1109/CDC.1975.270674.

5. Davison E, Goldenberg A. Robust control of a general servomechanism problem: The servo compensator. Automatica 1975; 11(5):461 - 471, doi:http://dx.doi.org/10.1016/0005-1098(75)90022-9. URL http://www.sciencedirect.com/science/article/pii/0005109875900229. 
6. Serrani A, Isidori A, Marconi L. Semi-global nonlinear output regulation with adaptive internal model. Automatic Control, IEEE Transactions on Aug 2001; 46(8):1178-1194, doi:10.1109/9.940923.

7. Marino R, Tomei P. Output regulation for linear systems via adaptive internal model. Automatic Control, IEEE Transactions on Dec 2003; 48(12):2199-2202, doi:10.1109/TAC.2003.820143.

8. Obregn-Pulido G, Castillo-Toledo B, Loukianov A. A structurally stable globally adaptive internal model regulator for mimo linear systems. Automatic Control, IEEE Transactions on Jan 2011; 56(1):160-165, doi: 10.1109/TAC.2010.2090409.

9. Bodson M, Douglas SC. Adaptive algorithms for the rejection of sinusoidal disturbances with unknown frequency. Automatica 1997; 33:2213-2221.

10. Nikiforov V. Adaptive non-linear tracking with complete compensation of unknown disturbances. European Journal of Control 1998; 4(2):132 - 139, doi:http://dx.doi.org/10.1016/S0947-3580(98)70107-4. URL http://www.sciencedirect.com/science/article/pii/s0947358098701074.

11. Lan W, M Chen B, Ding Z. Adaptive estimation and rejection of unknown sinusoidal disturbances through measurement feedback for a class of non-minimum phase non-linear mimo systems. International Journal of Adaptive Control and Signal Processing 2006; 20(2):77-97, doi:10.1002/acs.888. URL http://dx.doi.org/10.1002/acs.888.

12. Liu L, Chen Z, Huang J. Parameter convergence and minimal internal model with an adaptive output regulation problem. Automatica 2009; 45(5):1306 - 1311, doi:http://dx.doi.org/10.1016/j.automatica.2009.01.003. URL http://www.sciencedirect.com/science/article/pii/s0005109809000442.

13. Bobtsov A, Kolyubin S, Pyrkin A. Compensation of unknown multi-harmonic disturbances in nonlinear plants with delayed control. Automation and Remote Control 2010; 71(11):2383-2394, doi:10.1134/S000511791011010X. URL http://dx.doi.org/10.1134/S000511791011010x.

14. Bodson M. Rejection of periodic disturbances of unknown and time-varying frequency. International Journal of Adaptive Control and Signal Processing 2005; 19(2-3):67-88, doi:10.1002/acs.844. URL http://dx.doi.org/10.1002/acs.844.

15. Händel P, Tichavsky P, Savaresi SM. Large error recovery for a class of frequency tracking algorithms. International Journal of Adaptive Control and Signal Processing 1998; 12(5):417-436.

16. Carnevale D, Galeani S, Astolfi A. Hybrid observer for multi-frequency signals. IFAC Workshop Adaptation and Learning in Control and Signal Processing (ALCOSP), vol. 10, Elsevier (ed.), Antalya, 2010, doi: 10.3182/20100826-3-TR-4015.00011.

17. Carnevale D, Galeani S. On the tuning of a hybrid observer for multiple frequency estimation. Proc. 50th IEEE Conf. Decision and Control, Orlando, Florida, 2011; 6091-6096, doi:10.1109/CDC.2011.6160888.

18. Galeani S, Carnevale D, Astolfi A. An adaptive hybrid robust regulator. Proc. 50th IEEE Conf. Decision and Control, Orlando, Florida, 2011; 3350-3355, doi:10.1109/CDC.2011.6160893.

19. Kay SM, Marple SL. Spectrum analysis - a modern perspective. Proc. IEEE 1981; 69(11):1380-1419.

20. Regalia P. An improved lattice-based adaptive iir notch filter. IEEE Trans. Signal Processing Sept 1991; 39:21242128.

21. Bittanti S, Savaresi S. On the parameterization and design of an extended kalman filter frequency tracker. IEEE Trans. Automat. Contr. 2000; 45(9):1718-1715.

22. Hsu L, Ortega R, Damm G. A globally convergent frequency estimator. IEEE Trans. Autom. Contr. 1999; 44(4):698-713.

23. Sastry S, Bodson M. Adaptive Control: Stability, Convergence and Robustness. NJ, Prentice-Hall, 1989.

24. Xia X. Global frequency estimation using adaptive identifiers. IEEE Trans. Autom. Contr. 2002; 47(7):1188-1191.

25. Obregon-Pulido G, Castillo-Toledo B, Loukianov A. A globally convergent estimator for $\mathrm{n}$-frequencies. IEEE Trans. Autom. Contr. 2002; 47(5):857-863.

26. Marino R, Tomei P. Global estimation of n unknown frequencies. IEEE Trans. Autom. Contr. 2002; 47(8):1324 1328.

27. Carnevale D, Astolfi A. A minimal dimension observer for global frequency estimation. Proc. IEEE American Control Conference, Seattle, Washington, 2008; 5269-5274, doi:10.1109/ACC.2008.4587326.

28. Marino R, Santosuosso GL. Regulation of linear systems with unknown exosystems of uncertain order. IEEE Trans. Automatic Control feb 2007; 52(2):352 -359, doi:10.1109/TAC.2006.890376.

29. Hoagg J, Santillo M, Bernstein D. Discrete-time adaptive command following and disturbance rejection with unknown exogenous dynamics. Automatic Control, IEEE Transactions on may 2008; 53(4):912 -928, doi: 10.1109/TAC.2008.920234.

30. Goebel R, Sanfelice R, Teel AR. Hybrid dynamical systems. IEEE Contr. Sys. Magazine 2010; 29(2):28-93.

31. Zhou K, Doyle J, Glover K. Robust and optimal control. Prentice Hall Englewood Cliffs, NY, 1996.

32. Hespanha JP, Morse AS. Switching between stabilizing controllers. Automatica 2002; 38(11):1905 - 1917. 
33. Liberzon D. Switching in Systems and Control. Birkhäuser, 2003. 